\title{
The chemical abundances in a sample of dwarf irregular galaxies
}

\section{Small scale variations within NGC $6822 ?^{\star, \star \star}$}

\author{
A. M. Hidalgo-Gámez ${ }^{1}$, K. Olofsson ${ }^{1}$, and J. Masegosa ${ }^{2}$ \\ 1 Astronomiska observatoriet, Box 515, 75120 Uppsala, Sweden \\ e-mail: kjell.olofsson@astro.uu.se \\ 2 Instituto de Astrofísica de Andalucía, CSIC, Apdo. 3004, 18080 Granada, Spain \\ e-mail: pepa@iaa.es
}

Received 9 November 1999 / Accepted 18 October 2000

\begin{abstract}
Deep groundbased spectra of Hubble V and Hubble X, the two largest and brightest H II regions in the Local Group dwarf galaxy, NGC 6822, are presented. The extinction, excitation and ionization are studied on scales as small as $4 \mathrm{pc}$. The chemical composition of some elements within the regions as well as possible small scale variations in the abundances of helium, nitrogen, oxygen and neon over the face of the two regions are investigated. The detection of the emission lines [OI] and [CIIII] are probably related to shock-heating from gas ejected from supernova explosions and planetary nebula ejections, respectively, although the contribution to the total ionization is very small. No significant differences in chemical composition was found between Hubble $\mathrm{V}$ and Hubble X. However, some small scale variations in the abundances of oxygen, and possibly nitrogen and helium, over the face of both $\mathrm{H}$ II regions were noted, although different physical conditions could apply within the regions, such as local fluctuations in the temperature.
\end{abstract}

Key words. galaxies: evolution - galaxies: irregular - galaxies: stellar content - ISM: H II regions

\section{Introduction}

Much effort has been devoted to the study of the chemical abundances of dwarf irregular galaxies (dI) (e.g. Pagel \& Edmunds 1981; Ferguson et al. 1998). Most investigations focused on the measurement of the oxygen abundances of $\mathrm{H}$ II regions, with subsequent interpretation within the closed box model of chemical evolution (Tinsley 1978). It is usually assumed in these studies (Kinman \& Davidson 1981; Shield 1990; Dinerstein 1990) that the metallicity of the $\mathrm{H}$ II regions is homogeneous on both small and large scales.

In contrast to the spiral galaxies, where important gradients in the chemical abundances are found (e.g. Belley \& Roy 1992), the situation of the chemical homogeneity of the ISM in dI is still under debate. The absence of strong dynamical instabilities in dI, e.g. turbulent gas motions, make the mixing of the elements less efficient (Roy \& Kunth 1995). To add to the complexity, the internal dynamics and star formation triggering processes and

\footnotetext{
Send offprint requests to: A. M. Hidalgo-Gámez,

e-mail: anamaria@astro.uu.se

* Based on observations made at the European Southern Observatory, La Silla, Chile.

** Figures 2 and 5 are only available in electronic form at http://www. edpsciences.org
}

environments could imply a very different history of chemical evolution for each galaxy.

For a number of galaxies studied, the difference in the oxygen abundances comparing $\mathrm{HII}$ regions are smaller than the error in the measurements, e.g. DDO 50 (Masegosa et al. 1991) and NGC 2366 (González-Delgado et al. 1994), while for some others the differences are larger, e.g. IC 10 (Lequeux et al. 1979) and WLM (Hodge \& Miller 1995). One reason for the lack of agreement could be the relatively poor quality of the data used in the abundance determinations. This situation is greatly improved with the new observational facilities currently available and new studies of galaxies undergoing star formation, such as NGC 4214 (Kobulnicky \& Skillman 1996) and NGC 5253 (Kobulnicky et al. 1997), have been carried out. For these two galaxies, no inhomogeneities along the slit were found, and only a local enhancement of nitrogen was detected in NGC 5253.

This paper focuses on the distribution of chemical elements within two H II regions of the dI NGC 6822. Similar studies were carried out by Kobulnicky \& Skillman (1996) and Kobulnicky et al. (1997). The main difference between the latter studies and the present investigation is that, here, the metallicity determination has been limited to the locations in the $\mathrm{H}$ II regions where the emission 
line $[\mathrm{OIII}] \lambda 4363 \AA$ was detected, while in previous studies the determination of the abundances were extended to the whole region observed. For those locations where the [OIII] line was not detected, Kobulnicky et al. used an empirical method in the derivation of the oxygen abundances based on a typical value of the electron temperature. If the uncertainties associated with the abundances from the empirical methods are too high, no firm conclusions on possible abundance variations can be drawn. Another difference between the investigations by Kobulnicky \& Skillman (1996), Kobulnicky et al. (1997) and the one presented here, concerns the size of the galaxies involved. Both NGC 4214 and NGC 5253 have optical radii of 56 times larger than NGC 6822, the galaxy which is the subject of this investigation, and the size might play an important role in the chemical homogenization of the ISM. In order to bring more clarity to the problem of the presence or absence of chemical inhomogeneities, good quality data, such as is presented here, should be included in the investigation. Another investigation is presently being undertaken by the authors which includes some other dwarf galaxies, such as IC 4662 and IC 10.

NGC 6822 (DDO 209) is a Local Group dI with a barred structure (de Vaucouleurs et al. 1991). NGC 6822 was chosen because of its proximity to the Galaxy, which makes this object one of the best candidates for a thorough study of chemical composition. NGC 6822 is one of the best studied Local Group dI (e.g. Marconi et al. 1995; Gallart et al. 1996; Petitpas \& Wilson 1998; Cohen \& Blakeslee 1998). This galaxy is also the closest dI to the Milky Way at a distance of $0.48 \mathrm{Mpc}$ (Hidalgo-Gámez \& Olofsson 1998). The absolute magnitude is $M_{b}=-14.75$ and the optical radius is $1.08 \mathrm{kpc}$ (Hidalgo-Gámez \& Olofsson 1998). Recent observations at many different wavelengths have been performed; radio (Israel 1997), infrared (Gallagher et al. 1991), UV (Longo et al. 1991) and X-ray (Eskridge \& White 1997). A total of 157 H I r regions have been catalogued throughout the galaxy (Hodge et al. 1989).

The two largest and brightest H II regions, located at the northern part of this elongated galaxy, with only $320 \mathrm{pc}$ separating them, are the ones under study here: Hubble V and Hubble X. They have been selected because their large sizes and high surface brightnesses offer a unique opportunity to study possible variations in the chemical abundance of the elements within the $\mathrm{HII}$ regions. Even though they are the brightest regions in NGC 6822 they are not comparable in luminosity to the most powerful and bright $\mathrm{HII}$ regions observed such as the Orion nebula or 30 Dor. The $\mathrm{H} \alpha$ luminosity of Hubble $\mathrm{V}$ is $410^{38} \mathrm{erg} \mathrm{s}^{-1}$, which is only about $5 \%$ of the Orion complex (Hodge et al. 1989). These authors also determined the optical sizes, which are 112 pc for Hubble V and $143 \mathrm{pc}$ for Hubble X.

The oxygen abundance of these two H II regions have previously been determined by e.g. Peimbert \& Spinrad (1970), Lequeux et al. (1979), Pagel et al. (1980), Talent (1980), Skillman et al. (1989). In all these studies,
Table 1. Coordinates and position angles of the $\mathrm{H}$ II regions observed. The standard position angle of EFOSC1 is $270^{\circ}$

\begin{tabular}{cccc}
\hline H II region & $\alpha$ & $\delta$ & PA \\
\hline Hubble V & $19^{\mathrm{h}} 44^{\mathrm{m}} 57^{\mathrm{s}}$ & $-14^{\circ} 43^{\prime} 41^{\prime \prime}$ & $270^{\circ}$ \\
Hubble X & $19^{\mathrm{h}} 44^{\mathrm{m}} 48^{\mathrm{s}}$ & $-14^{\circ} 44^{\prime} 30^{\prime \prime}$ & $270^{\circ}$ \\
\hline
\end{tabular}

relatively old instrumentation was used. Even though the mean chemical abundances of many elements of Hubble $\mathrm{V}$ and Hubble $\mathrm{X}$ agree well with the ones determined in those investigations (see Sect. 5.4), the older instrumentation could not allow spatially resolved studies of the abundances.

Because of the good quality of the data presented here, a two-dimensional mapping of Hubble $\mathrm{V}$ and Hubble $\mathrm{X}$ of regions of $4 \mathrm{pc}$ could be performed and therefore details on small scales could be resolved. Apart from a determination of the elemental abundances of the two regions, a mapping of the extinction, excitation and ionization throughout both regions is possible, as well as a study of the spatial distribution of chemical elements throughout the regions. This concerns in particular nitrogen and oxygen which are the elements with the most reliable data.

The next section is devoted to the description of the acquisition of the data and its reduction. In Sect. 3 the analysis is outlined. Section 4 is devoted to the extinction determination as well as the correction for underlying stellar absorption in the spectra. The results and discussions are presented in Sect. 5. Conclusions are presented in Sect. 6.

\section{Observations}

Continuum-subtracted $\mathrm{H} \alpha$ images of Hubble $\mathrm{V}$ and Hubble $\mathrm{X}$ are presented in Figs. 1a and b. The observations were carried out on the 10th of August, 1997 with the ESO $3.6 \mathrm{~m}$ telescope at La Silla, Chile. EFOSC1 was used to obtain spectra as well as $\mathrm{H} \alpha$ and continuum images. The coordinates for the spectroscopical slit position centers and position angles are given in Table 1. The images were used as an aid for positioning the slit. A $512 \times 512$ pixel CCD (No. 26 in the ESO-listing) was used as a detector. The total field-of-view of the CCD is $5 ! 2 \times 5 ! 2$ and the pixel size is $27 \mu \mathrm{m}$. Two grisms were used for the observations. They covered a range in wavelength between $3720-5530 \AA$ in the blue (grism 6) and 5150-6900 $\AA$ in the red (grism 10). The resolution was $8 \AA$ for both grisms. The original starting wavelength of the grism of the blue spectral regions was $3740 \AA$ and, in order to include the forbidden emission line [OII] $\lambda 3727 \AA$, a displacement towards the blue was introduced which resulted in a central wavelength of $4500 \AA$. The slit, with a width of 1 ". 5 (in order to match the seeing conditions) and a length of 3!' for the blue (the movable slit) and 3!9 for the red, passed through the HII regions in the E-W direction. For the standard stars the slit was opened to 

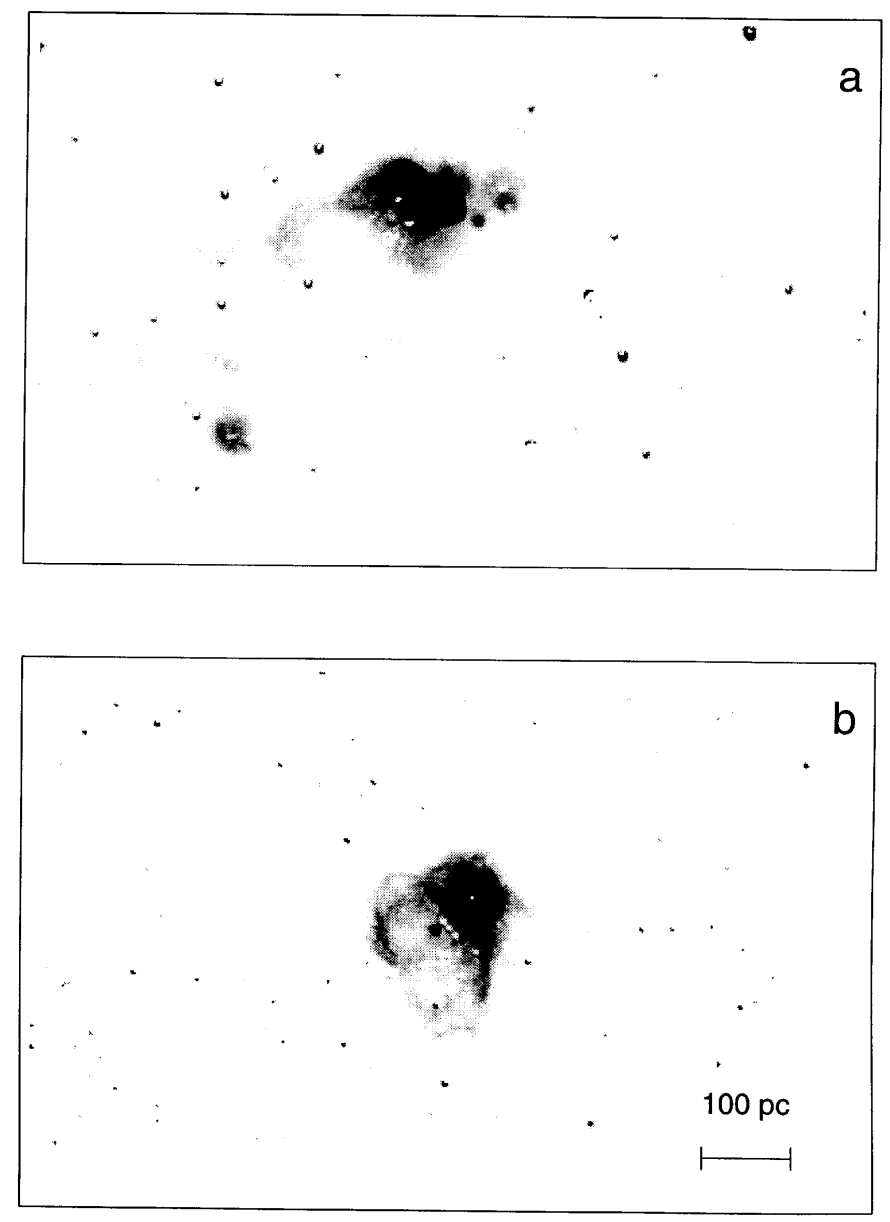

Fig. 1. The continuum-subtracted $\mathrm{H} \alpha$ image of a) Hubble $\mathrm{V}$ and $\mathbf{b}$ ) Hubble $\mathrm{X}$. North is up and east to the right

$5^{\prime \prime}$. The total integration time for Hubble $\mathrm{V}$ was $50^{\mathrm{m}}$ in the blue and $40^{\mathrm{m}}$ in the red and for Hubble X, $40^{\mathrm{m}}$ and $30^{\mathrm{m}}$, respectively. For the target objects, the blue and red spectral regions were observed consecutively in order to ensure that the same location in each $\mathrm{H}$ II region was observed. Two subexposures were obtained in order to check for cosmic events. The sky was clear at the zenith position but there were some clouds just above the horizon. The seeing conditions were stable throughout the night, in particular during the observations of NGC 6822 with a seeing of $1^{\prime \prime} 25$. The airmasses were 1.22 and 1.09 for the blue and the red, respectively, for Hubble V. The corresponding values for Hubble $\mathrm{X}$ were 1.03 and 1.06. The observations were performed outside the paralactic angle. Due to the rather low air masses, small, or no corrections for differential refraction were applied. It was found, following Table 2 of Fillipenko (1982), that the loss of light in the vicinity of $[\mathrm{OII}] \lambda 3727 \AA$ was $2 \%$. In such cases, corrections were made.

The data was reduced with the software package MIDAS. Bias and flat-fielding were performed in the standard way, with bias-frames and continuum lamps. Considering the negligible changes in the flat frames over several consecutive nights, a single flat frame for each
Table 2. The maximum uncertainties in the intensities of the emission lines of the $3 \mathrm{r}$-spectra. These uncertainties correspond to the spectra at the edges of the $\mathrm{H}$ iI regions

\begin{tabular}{ccc}
\hline $\begin{array}{c}\text { Line } \\
(\AA)\end{array}$ & $\begin{array}{c}\text { Hubble V } \\
(\%)\end{array}$ & $\begin{array}{c}\text { Hubble X } \\
(\%)\end{array}$ \\
\hline$[\mathrm{OII}] \lambda 3727$ & 61 & 41 \\
{$[\mathrm{NeIII}] \lambda 3869$} & 53 & 44 \\
$\mathrm{HeI} \lambda 3889$ & 81 & 47 \\
{$[\mathrm{NeIII}] \lambda 3970$} & 75 & 50 \\
$\mathrm{H} \delta \lambda 4102$ & 73 & 68 \\
$\mathrm{H} \gamma \lambda 4340$ & 64 & 19 \\
{$[\mathrm{OIII}] \lambda 4363$} & 75 & 85 \\
$\mathrm{HeI} \lambda 4471$ & 67 & 68 \\
$\mathrm{H} \beta \lambda 4861$ & 15 & 12 \\
{$[\mathrm{OIII}] \lambda 4959$} & 22 & 33 \\
{$[\mathrm{OIII}] \lambda 5007$} & 8 & 6 \\
$\mathrm{HeI} \lambda 5875$ & 81 & 49 \\
{$[\mathrm{NII}] \lambda 6548$} & 94 & 94 \\
$\mathrm{H} \alpha \lambda 6563$ & 16 & 6 \\
{$[\mathrm{NII}] \lambda 6583$} & 29 & 91 \\
$\mathrm{HeI} \lambda 6678$ & 40 & 42 \\
{$[\mathrm{SII}] \lambda 6716$} & 67 & 29 \\
{$[\mathrm{SII}] \lambda 6730$} & 80 & 41 \\
\hline
\end{tabular}

grism was used, averaging a total of 19 and 23 flat-frames for the blue and red spectral regions, respectively. No sky flats were performed and the spectral response of the CCD was corrected by averaging the values in the spatial direction. The final frames, obtained by dividing the normalized flat-fields by the averaged ones, were completely flat along the spatial direction but with some small structures in the spectral direction, even though the median was 1.00 in all cases. All the flat-frames were observed through the $1^{\prime \prime} .5$ slit. He-Ar lamps were used for the wavelength calibration. Due to the long exposures needed in order to obtain a high signal-to-noise ratio, $2-3$ arc-frames were obtained during each night, with different inclinations in order to check for flexures. No flexures were found along the lines, nor in the arc-frames or in the sky lines observed in the spectra. The calibrations resulted in rms of 0.28 and 0.40 using the blue and red grisms, respectively. The sky subtraction was performed using the batch in MIDAS, where four points were needed. The method defined two regions of the sky, normally one at each side of the emission region. Hubble $\mathrm{V}$ was quite well centered on the slit and there was a sufficient number of pixels for an accurate sky subtraction. In the case of Hubble X, a displacement was evident towards one edge of the slit and only 20 pixels were available near this edge. In any case, due to the large number of pixels at the other edge, an acceptable sky subtraction could be performed. Only the sky lines located near $6300 \AA$ remained. The flux calibrations were 
performed with spectra of the standard stars LTT 1788 and LTT 1020. The accuracy of the calibrations was $5 \%$ in the blue and $8 \%$ in the red spectral region. The atmospheric extinction correction was made using the tables of La Silla.

\section{Analysis of the data}

\subsection{The handling of the data}

One of the main goals of this paper is to study the spatial distribution of chemical elements within the H II regions. The two-dimensional spectra (Figs. 2a and b, only available electronically) were divided into three-row spectra (hereafter, $3 r$-spectra). The spatial resolution of three pixels corresponds to a seeing of about 1.5 , since the pixel size is 0. "61/pixel for EFOSC1. A 3r-spectrum corresponds to a linear scale of $4.3 \mathrm{pc}$ at the distance of NGC 6822. The total number of 3r-spectra covering the $\mathrm{H}$ II regions was 44 for the blue and 54 for the red spectral regions of Hubble $\mathrm{V}$. The corresponding numbers for Hubble $\mathrm{X}$ were 34 and 33 . The number of $3 \mathrm{r}$-spectra are based on different lines in each spectral range, $\mathrm{H} \alpha$ in the red and $[\mathrm{OII}] \lambda 3727 \AA$ in the blue, which are, normally, the most conspicuous emission lines in this type of object. A crucial task is to ascertain that, using both grisms, the pointing is towards the same location within the $\mathrm{H}$ II regions. The alignment of both the blue and the red was performed with the aid of the continuum between $5200 \AA$ and $5400 \AA$ which is the range where the blue and the red regions overlap. A displacement of 15 pixels resulted for Hubble $\mathrm{V}$ between the blue and the red. Even though the overlapping was not perfect this resulted in a deviation of less than one pixel. The situation is more favourable for Hubble $\mathrm{X}$ where a displacement of only one pixel was necessary in order to align both the and the red. The problem with overlap is quite common when one-arm spectrographs are used, such as EFOSC1. This is due to small movements of the observational setup (instrument+CCD+telescope) between the observations using the blue and the red grisms.

The faintest lines were considered real detections when the intensity was $1 \sigma$ higher than the average noise level in the vicinity of the line. Moreover, in order to estimate the uncertainties due to the determination of the continuum level and to avoid misidentification of noisy peaks with weak lines, each 3r-spectrum was analysed several times using several different continuum levels. Only spectral lines which were detected in two of these were considered real detections.

At the resolution of $8 \AA$ it was found that some of the emission lines were blended, especially $[\mathrm{SII}] \lambda \lambda 6716,6730 \AA,[\mathrm{NII}] \lambda 6548 \AA$ with $\mathrm{H} \alpha$ as well as $[\mathrm{OI}] \lambda 6300 \AA$ with $[\mathrm{SIII}] \lambda 6312 \AA$. For the blended lines a special routine in the software was used which fits two Gaussian profiles to the doublet to provide the intensity of each line. These intensities were compared with those obtained from the transition probability between lines of
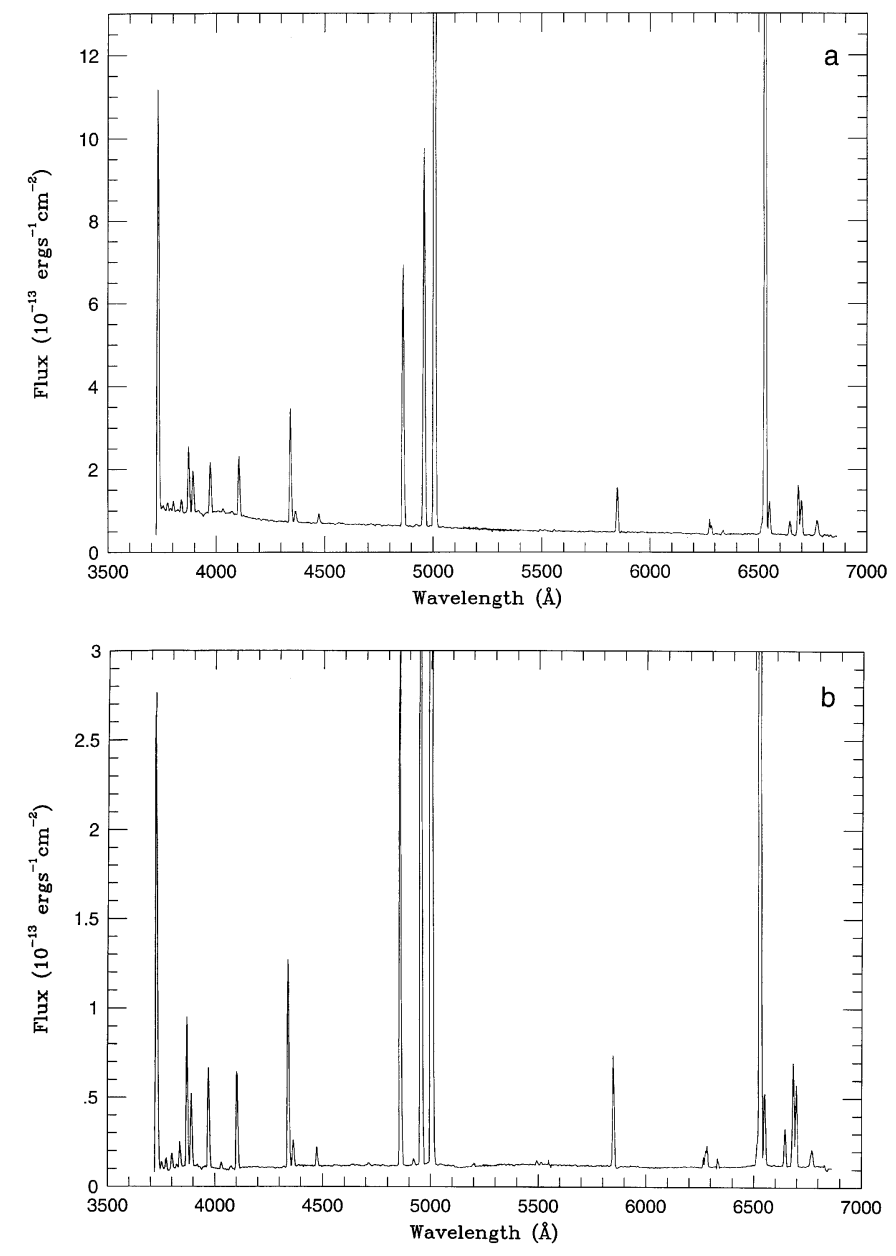

Fig. 3. The spatially-averaged spectrum of a) Hubble V and b) Hubble $\mathrm{X}$. The figures are scaled in order to emphasize weaker spectral features

the same ionization stage and the total intensity of the doublet. Differences in the intensity values were added to the uncertainties (see Sect. 3.2). In most cases, the agreement was good. A set of intensities, normalized to the $\mathrm{H} \beta$ emission line, for each $3 \mathrm{r}$-spectrum was finally obtained. Due to a difference of half a pixel between the blue and the red spectral regions of Hubble $\mathrm{V}$, the red region of each 3 r-spectrum was normalized to the $\mathrm{H} \alpha$ line intensities.

Another point of interest is how to derive the average metallicity for each $\mathrm{HII}$ region. In order to reach an average, new spectra were obtained by summing all the rows where the emission from the forbidden line [OIII] $\lambda 4363 \AA$ was detected. These are named spatiallyaveraged spectra, and refer to the total nebular emission from each $\mathrm{H}$ II region within the slit. They correspond to a total of 51 rows for Hubble V and 33 for Hubble X, which give total physical sizes of $215 \mathrm{pc}$ and $139 \mathrm{pc}$, respectively. The spatially-averaged spectra are presented in Figs. 3a and $b$. 


\subsection{Errors in the continuum and line intensities}

For any conclusive answer regarding possible variations in the chemical abundance in $\mathrm{H}$ II regions, a careful estimate of the uncertainties of the continuum and line intensities is of the utmost importance. In this analysis, different sources of uncertainty have been considered: uncertainties in the level of the spectral continuum with respect to the line, uncertainties due to the different corrections (in particular the extinction correction), uncertainties introduced by the reduction procedure and uncertainties due to the blending of spectral lines. The uncertainty introduced by photon counting was found to be very small in the parts of the spectra devoted to the analysis. This is due to the low level of noise and this possible source of error is therefore neglected.

It was noticed that the major contributor to the uncertainty for each 3r-spectrum was the determination of the continuum level. The uncertainties were estimated using the dispersion of the raw intensity of each line. Even though the uncertainties associated with the extinction correction were important and accounted for in the error analysis, they were in most cases smaller than the uncertainties associated with the continuum.

The reduction procedure can also introduce uncertainties. From our analysis it has been estimated that an important source of uncertainty in the resulting intensities is the deviation of the response curve of the standard star from the observed flux data. The deviations were estimated to be $8 \%$ in the vicinity of the $\mathrm{H} \beta$ line, $4 \%$ close to [OII $] \lambda 3727 \AA$ and the $[\mathrm{OIII}] \lambda 5007 \AA$ line and $20 \%$ close to $[\mathrm{SII}] \lambda 6716 \AA$.

For the blended lines an additional error term was added. In this case, the lines were deblended by a special routine in the software. This term takes into account the goodness of the deblending procedure performed by the software. Uncertainties of $49 \%$ for $[\mathrm{NII}] \lambda 6548 \AA, 16 \%$ for [SII] $\lambda 6716 \AA$ and $36 \%$ for $[\mathrm{SII}] \lambda 6730 \AA$ were obtained for Hubble V. The corresponding uncertainties for Hubble X were $54 \%$ for $[\mathrm{NII}] \lambda 6548 \AA, 6 \%$ for $[\mathrm{SII}] \lambda 6716 \AA$ and $9 \%$ for $[\mathrm{SII}] \lambda 6730 \AA$.

All these uncertainties were summed for each spectrum. Table 2 presents the error percentage of the emission lines for each region. It should be noted that these numbers correspond to those 3r-spectra with the lowest signal-to-noise ratio in the emission line [OIII] $\lambda 4363 \AA$. Therefore, they must be considered as the maximum statistical errors affecting the $3 r$-spectra. These spectra contain, mainly, the outer part of the $\mathrm{H}$ II regions and the errors at the central parts, which will be the regions under consideration in this investigation (see Sect. 5.3), are smaller.

Systematic errors are much more difficult to assess. These should, in particular, be introduced during observation. A possible source of error is bad columns on the CCD. No evidence of systematic errors was found during the course of this work although systematic errors cannot be completely ruled out.

\section{The correction for extinction and underlying stellar absorption}

In order to derive the abundances of the elements from the intensities of the lines, two main corrections must be applied: the spectra must be corrected for underlying stellar absorption and extinction.

\subsection{The correction for underlying stellar absorption}

The absorption features arising from the stellar component can severely affect some emission line intensities. The absorption affects, mainly, the Balmer lines but other lines are also affected. In this analysis, an elaborate absorption correction was applied. The line intensities were corrected using the expression of McCall et al. (1985)

$\log I(\lambda)=\log I_{\mathrm{o}}(\lambda)+\frac{A_{\mathrm{v}, \mathrm{c}}}{8}[f(\lambda)-f(\mathrm{H} \beta)]-\log \left(1+\frac{E_{v}}{e_{\beta}}\right)$

where $I_{\mathrm{o}}(\lambda)$ is the normalized intensity, $f(\lambda)$ is the extinction law, $E_{\mathrm{v}}$ is the emission equivalent width of the line used in the reddening correction, $e_{\beta}$ is the absorption equivalent width of $\mathrm{H} \beta$ and $A_{\mathrm{v}, \mathrm{c}}$ is a coefficient defined as

$$
A_{\mathrm{v}, \mathrm{c}}=\frac{-8.0}{[f(\lambda)-f(\mathrm{H} \beta)]} \log \left[\frac{1+\frac{E_{\mathrm{v}}}{e(\lambda)}}{1+\frac{E_{\mathrm{v}}\left(\mathrm{H}_{\beta}\right)}{e_{\beta}}}\right]
$$

where $E_{\mathrm{v}}(\mathrm{H} \beta)$ is the emission equivalent width of $\mathrm{H} \beta$ and $e(\lambda)$ the absorption equivalent width of the Balmer lines. The emission equivalent widths of the Balmer lines are obtained from spectra. A synthetic model of spectral evolution (Olofsson 1995), with a metallicity of $40 \%$ of the solar value and an age of a single burst of star formation of $6 \mathrm{Myr}$, was used for the absorption values. Star formation under the assumption of a Salpeter initial mass function (Salpeter 1955) was assumed. The model was chosen according to the metallicity values available in the literature (e.g. Pagel et al. 1980) and the ages of Hubble V and Hubble X determined by Wilson (1992) (see Sect. 5.3.2). This approach is more preferable than direct measurements, due to the weakness of the absorption features in the $3 \mathrm{r}$ spectra, especially in the $\mathrm{H} \gamma$ line (the line used for the reddening correction in Hubble $\mathrm{V}$, see Sect. 4.2). When measurable, typical values of $2-3 \AA$ were obtained.

Following the absorption correction a new normalization to the $\mathrm{H} \beta$ line and a reddening correction was applied.

\subsection{The extinction correction}

Besides the dust within the Galaxy, which has not been corrected for in this analysis since this is only a matter of scaling, dust could reside within the emission region itself. In addition, dust may be uniformly distributed outside the nebula but within NGC 6822, and made up of clumpy dust grains (Caplan \& Deharveng 1986). Since the extinction is wavelength dependent, it will affect the relative intensities of the lines and for an appropriate determination of the 
physical properties of the $\mathrm{H}$ II regions, a correction for this effect should be applied. There are two different methods at hand. The first is based on a comparison of the intensities from the radio continuum and e.g. the optical $\mathrm{H} \beta$ line. Since the extinction is negligible in the radio wavelength region, the total extinction in the $\mathrm{H} \beta$ line is obtained directly (see e.g. Skillman 1984; Masegosa 1988 for details). The second method is based on the Balmer emission line decrement. The strength of the Balmer lines depends only weakly on the electron temperature $\left(T_{\mathrm{e}}\right)$, thus the ratio between observed and theoretical line intensities gives a measurement of the amount of extinction.

In this investigation, the Balmer decrement is used for the extinction correction. The expression used was

$C_{\beta}=-(f(\lambda)-f(\mathrm{H} \beta))^{-1} \ln \frac{\left(I\left(\lambda_{\mathrm{o}}\right) / I\left(\mathrm{H} \beta_{\mathrm{o}}\right)\right)}{(I(\lambda) / I(\mathrm{H} \beta))}$

where $C_{\beta}$ is the extinction coefficient in $\mathrm{H} \beta$ and $f(\lambda)$ is the extinction law. $I\left(\lambda_{\mathrm{o}}\right) / I\left(\mathrm{H} \beta_{\mathrm{o}}\right)$ is the observed intensity of the line relative to $\mathrm{H} \beta$ and $I(\lambda) / I(\mathrm{H} \beta)$ is the theoretical value determined for an optically thick gas (Brocklehurst 1971). The most often used extinction laws are the ones of Whitford (1958) and the Whitford modified law (Savage \& Mathis 1979), although some others have been proposed (e.g. Costero \& Peimbert 1970; Schild 1977). As has been claimed, the difference in the intensity of the lines as a result of the use of different extinction laws is usually not significant compared to the uncertainties associated with the observations (McCall et al. 1985; Masegosa 1988). In this study the Whitford modified law was used.

Due to the overlapping problem in the spectra of Hubble V mentioned previously (see Sect. 3.1), the extinction coefficient was determined from the intensity of the $\mathrm{H} \gamma$ line instead of the $\mathrm{H} \alpha$ line. Following the reddening correction of the lines, the red part was normalized by the theoretical value of the ratio $\mathrm{H} \alpha / \mathrm{H} \beta$. Case $\mathrm{B}$ recombination was assumed with an $T_{\mathrm{e}}$ of $10000 \mathrm{~K}$, which is close to the $T_{\mathrm{e}}$ determined with the use of the oxygen lines for both regions. For Hubble X, the extinction coefficient was determined using the $\mathrm{H} \alpha$ line.

As Caplan \& Deharveng (1986) pointed out, the best way of distinguishing between internal and external extinction is by comparing the extinction derived from the optical Balmer decrement and the radio wavelength region. The latter part of the spectrum is evidently not attainable in this investigation. In order to obtain some clues about the location of the dust in the two $\mathrm{H}$ II regions, the $C_{\beta}$, based on the $\mathrm{H} \gamma$ line, and the $\mathrm{H} \alpha / \mathrm{H} \beta$ emission line ratio, normalized to their corresponding maxima, are compared (Figs. 4a and b for Hubble V and X, respectively). The $C_{\beta}$ derived from the $\mathrm{H} \gamma$ line is preferred since the $C_{\beta}$ based on the $\mathrm{H} \alpha$ line mimics the behaviour of the $\mathrm{H} \alpha$ intensity, independent of the origin of the extinction. Although only a limited amount of information could be extracted, some conclusions could be drawn. While the $\mathrm{H} \alpha / \mathrm{H} \beta$ ratio is approximately constant throughout Hubble $\mathrm{V}$, the $C_{\beta}$ varies. The same is true for Hubble X. This is an indication of external extinction because, if the
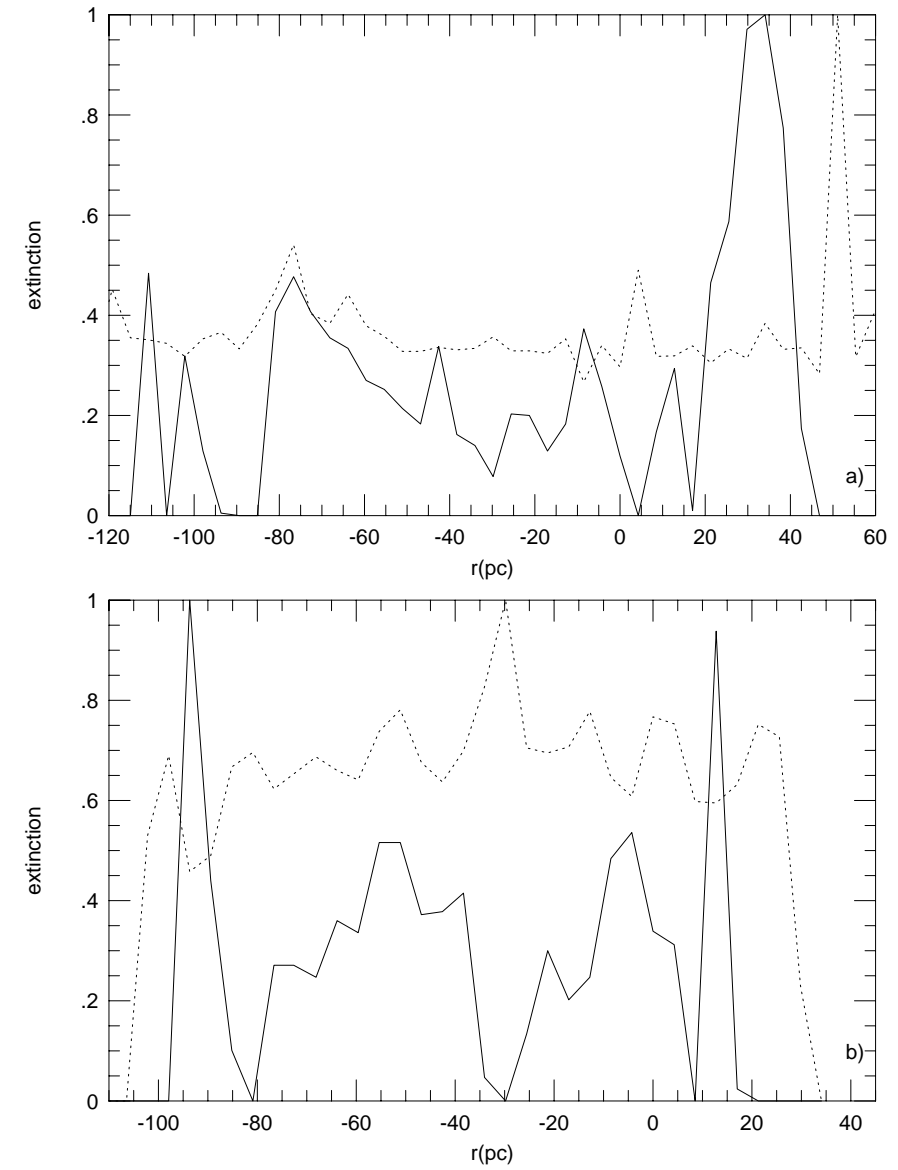

Fig. 4. a) The extinction across the face of Hubble V. The extinction coefficient, $C_{\beta}$ (-), based on the $\mathrm{H} \gamma$ line is shown, as well as the non reddening-corrected $\mathrm{H} \alpha / \mathrm{H} \beta$ emission line ratio $(\cdots)$. East is to the left. The high peaks at the edges are probably an effect of the poor signal-to-noise ratio in the outer parts of the H II region. b) The same for Hubble X. The extinction coefficient, $C_{\beta}(-)$, based on the $\mathrm{H} \alpha$ line, is visualized in this case

extinction would be internal to the nebula, these two parameters should follow a similar behaviour. It must be pointed out that the zero values in the $C_{\beta}$ in Figs. $4 \mathrm{a}$ and $\mathrm{b}$ are mainly due to the fact that, for these spectra, no extinction correction was made due the low signalto-noise ratio. Therefore, the zero points for the extinction are arbitrary. From the figures, it is also possible to draw some conclusions about the distribution of the dust. The situation is quite similar in both regions, with nondistinct clumps present in Hubble $\mathrm{X}$ and a big clump of dust throughout most of the eastern part of Hubble V.

Another indicator of internal extinction is the possible correlation between the emission line ratio [OIII]/[OII] and $C_{\beta}$ (González-Delgado et al. 1994) because the dust could be mixed with the ionized gas. Such a correlation does not exist, either for Hubble V or for Hubble X. The linear correlation coefficients are found to be $r_{1}=0.05$ and $r_{1}=0.01$, respectively, which confirms the external origin of the reddening. 
Table 3. Extinction coefficients, $C_{\beta}$, derived from the Balmer lines of the spatially-averaged spectra with non-corrected line intensities

\begin{tabular}{ccc}
\hline line & Hubble V & Hubble X \\
\hline $\mathrm{H} \alpha$ & $2.0 \pm .4$ & - \\
$\mathrm{H} \gamma$ & $1.92 \pm .04$ & $1.16 \pm .07$ \\
$\mathrm{H} \delta$ & $2.05 \pm .05$ & $0.99 \pm .01$ \\
$\mathrm{H} 9$ & $5.21 \pm .07$ & $5.08 \pm .01$ \\
$\mathrm{H} 10$ & $5.28 \pm .08$ & $4.84 \pm .01$ \\
$\mathrm{H} 11$ & $5.23 \pm .08$ & $3.22 \pm .01$ \\
$\mathrm{H} 12$ & $5.03 \pm .08$ & $5.82 \pm .02$ \\
\hline
\end{tabular}

The extinction coefficients for all the Balmer lines in both regions were determined from the spatially-averaged spectra. The results are presented in Table 3. There are some indications of decreasing values with increasing wavelengths. However, some exceptions, especially for $C_{\beta}$, based on the $\mathrm{H} \delta / \mathrm{H} \beta$ ratio in Hubble $\mathrm{X}$, are seen. The increasing values of $C_{\beta}$ toward shorter wavelengths is probably a result of underlying stellar absorption and cannot be explained by the uncertainties in the measurements, which are much smaller.

The colour excess $E(B-V)$ (de Vaucouleurs et al. 1991) determined in this investigation for the two H II regions correspond to $1.8 \mathrm{mag}$ and $2.5 \mathrm{mag}$, respectively, in the $B$-band. These rather high values indicate the presence of a dust component external to the regions but internal to the galaxy. The absorption within the Galaxy in the $B$-band is only $0.86 \mathrm{mag}$. The $E(B-V)$ values derived by Wilson (1992) and Marconi et al. (1995) agree with the high values obtained here, but do not agree with the $E(B-V)$ values obtained from previous spectroscopical investigations.

We emphasize the use of the $\mathrm{H} \gamma$ emission line in the reddening correction of Hubble $\mathrm{V}$ instead of $\mathrm{H} \alpha$ due to the lack of a perfect overlapping between the red and the blue part of the spectrum. It is well known that the $\mathrm{H} \gamma$ line is more affected by underlying stellar absorption, but comparing the values of the extinction coefficients (Table 3) determined with all the Balmer lines observed, no significant differences are obtained, with the exception of spectral lines with wavelengths shorter than $\mathrm{H} \delta$.

All results presented in the following sections include error estimates associated with the reddening correction.

\section{Results and discussion}

\subsection{The ionic chemical abundances}

Only those 3r-spectra for which the emission line [OIII] $\lambda 4363 \AA$ was detected were used in the determination of the chemical abundances. In Sect. 5.3 a discussion of the limits of the signal-to-noise ratio is presented. The total number of 3r-spectra was 17 for Hubble $\mathrm{V}$ and 16 for Hubble X. A five-level atom two-zone model was used to derive the chemical abundances (Bergvall 1999). The $T_{\mathrm{e}}$ in the high ionization zone $\left(\mathrm{O}^{++}\right)$is estimated using an iterative process based on the [OIII] line ratios (Osterbrock 1989). This method is often referred to as the temperaturesensitive method. The temperature of the low ionization zone $\left(\mathrm{O}^{+}\right)$was estimated using the relation of Vila-Costas \& Edmunds (1993).

The output from the model are the $T_{\mathrm{e}}$ of the high and low ionization zones of the nebula, the ionic abundances of the lines as well as the total oxygen abundance and the abundance ratio $\log (\mathrm{N} / \mathrm{O})$. Also, the oxygen abundance from semiempirical methods (Skillman 1989; McGaugh 1994) are provided for comparison. The electron density, $N_{\mathrm{e}}$, of the regions is determined from the ratio of the lines $[\mathrm{SII}] \lambda \lambda 6716,6730 \AA$. In cases of a poor signal-to-noise ratio in these lines, or if the lines where not properly resolved, the $N_{\mathrm{e}}$ was assumed to be $100 \mathrm{~cm}^{-3}$. This value is typical for this class of objects.

No error estimates were used in the derivation of these quantities; instead an alternative approach was made. The intensity of each line was increased by its error percentage. With these updated intensities, new ionic abundances were obtained and the uncertainties were estimated from the difference between the two values.

It may appear that applying a two-zone ionization model to individual $\mathrm{HII}$ regions of less than $5 \mathrm{pc}$ in size could give unrealistic results, since in the most unfavourable cases, a large portion of the ionization zone could reside outside the region considered. This would of course result in erroneous emission line ratios. As a check, new frames covering the pixels corresponding to four individual consecutive spectra, randomly chosen, for Hubble V and Hubble X, respectively, were obtained and two new sets of intensities were derived, as previously described (see Sect. 3). The chemical abundances were compared with the average values of the abundances derived from the four individual spectra. While the average oxygen abundance is $12+\log (\mathrm{O} / \mathrm{H})=8.16$ for Hubble $\mathrm{V}$, the oxygen abundance derived from the individual spectra is 8.20. For Hubble $\mathrm{X}$, the value is 8.10 in both cases. These results indicate that the differences are smaller than the errors in the abundance determinations.

\subsection{The derivation of the atomic chemical abundances}

The atomic abundances of the elements are derived from the ionic ones and the ionization correction factors (ICFs) which take into account the non-observable ions for each element.

For some elements, such as oxygen, no correction needs to be applied since all important ionization stages are within the wavelength range studied.

\subsubsection{Helium}

The ICF for helium is somewhat problematic since quite contradictory results exist. Izotov et al. (1997) proposed 
that no ICF was necessary for helium. On the other hand, Pagel et al. (1992) proposed the following expression

$\mathrm{ICF}=1+\eta(0.005+0.001 \eta)$

with $\eta=\frac{\mathrm{O}^{+}}{\mathrm{S}^{+}} \frac{\mathrm{S}^{++}}{\mathrm{O}^{++}}$(Vílchez \& Pagel 1988). The [SIII] abundances could be obtained from the intensity of the line [SIII] $\lambda 6312 \AA$, assuming the temperature for this region to be the same as that for the $\mathrm{O}^{++}$region. One problem is that this line is blended with [OI] $\lambda 6300 \AA$ and could affect the ionic abundance as well as the ICF. Another flaw is the absence of the lines at [SIII] $\lambda 9069,9532 \AA$ in the spectra used in this analysis. Even though a determination of the intensity of [SIII] $\lambda 6312 \AA$ could easily be obtained, the uncertainties imposed using this line would not allow an accurate determination of the ICF. As a consequence, no ICF was applied in the determination of the helium abundance of the objects.

\subsubsection{Nitrogen}

The most commonly used ICF for nitrogen is $N(\mathrm{O}) / N\left(\mathrm{O}^{+}\right) \quad$ (Torres-Peimbert \& Peimbert 1977) because of the affinity of the ionization potential between the two species, and also because of the lack of more highly ionized nitrogen in $\mathrm{H}$ II regions when only photoionization prevails. Garnett (1989) studied the applicability of this ICF and concluded that it could be applied to regions with metallicities lower than that of the LMC $(12+\log (\mathrm{O} / \mathrm{H})=8.3)$.

\subsubsection{Neon}

A derivation of the total abundance of neon can also be problematic since, in this investigation, only $\mathrm{Ne}^{++}$ appears in the wavelength region observed. The ICF proposed is based on the proximity of the ionization potentials between neon and oxygen and is given by

$\mathrm{ICF}=\frac{N(\mathrm{O})}{N\left(\mathrm{O}^{++}\right)}$

(Peimbert \& Torres-Peimbert 1977). The main problem is that for low excitation objects, this ICF predicts an underestimate of the (Ne/O) ratio (Masegosa et al. 1994). In order to clarify this situation a correlation between the $\mathrm{Ne}^{++}$and the $\mathrm{O}^{+}$abundances was obtained for both Hubble $\mathrm{V}$ and Hubble X. The linear correlation coefficients were $r_{1}=0.10$ and $r_{1}=0.17$, respectively, and it was therefore concluded that the suggested ICF could be applied.

\subsection{The spatial distribution}

In order to check for the reliability of the results, the signal-to-noise ratio of each 3r-spectrum are plotted in Figs. 5a and b (only available electronically). The signalto-noise ratio of the three emission lines, $\mathrm{H} \alpha, \mathrm{H} \beta$ and $[\mathrm{OIII}] \lambda 4363 \AA$, follows a similar behaviour and only the
Table 4. Line intensities, normalized to $\mathrm{H} \beta$, and total error estimates of Hubble $\mathrm{V}$ and Hubble $\mathrm{X}$, averaged over the whole of each region. The flux in the $\mathrm{H} \beta$ line is given in units of $\mathrm{erg} \mathrm{s}^{-1} \mathrm{~cm}^{-2}$

\begin{tabular}{|c|c|c|c|c|c|}
\hline Ion & $\lambda(\AA)$ & Hubble V & $( \pm)$ & Hubble X & $( \pm)$ \\
\hline$\left[\begin{array}{ll}\mathrm{O} & \mathrm{II}\end{array}\right]$ & 3727 & 1.6 & .1 & 2.3 & .1 \\
\hline H12 & 3750 & 0.020 & .001 & 0.015 & .001 \\
\hline H11 & 3771 & 0.027 & .001 & 0.041 & .008 \\
\hline H10 & 3798 & 0.040 & .002 & 0.039 & .003 \\
\hline He I & 3819 & 0.007 & .001 & 0.008 & .006 \\
\hline H9 & 3835 & 0.063 & .002 & 0.057 & .006 \\
\hline$[\mathrm{Ne} \mathrm{III}]$ & 3869 & 0.376 & .007 & 0.338 & .010 \\
\hline $\mathrm{He} \mathrm{I}+\mathrm{H} 8$ & 3889 & 0.189 & .004 & 0.207 & .008 \\
\hline$[\mathrm{Ne}$ III $]$ & 3970 & 0.268 & .003 & 0.258 & .008 \\
\hline \multicolumn{6}{|l|}{$+\mathrm{H} 7$} \\
\hline Fe I & 4002 & - & - & 0.005 & .002 \\
\hline He I & 4026 & 0.016 & .001 & 0.013 & .001 \\
\hline$[\mathrm{S}$ II $]$ & $\begin{array}{c}4068 \\
+4076\end{array}$ & 0.009 & .001 & 0.012 & .006 \\
\hline $\mathrm{H} \delta$ & 4101 & 0.253 & .002 & 0.280 & .006 \\
\hline $\mathrm{H} \gamma$ & 4340 & 0.469 & .005 & 0.49 & .01 \\
\hline [O III] & 4363 & 0.055 & .003 & 0.048 & .001 \\
\hline He I & 4471 & 0.039 & .001 & 0.037 & .003 \\
\hline$[\mathrm{Fe} \mathrm{III}]$ & 4658 & 0.002 & - & - & - \\
\hline \multicolumn{6}{|l|}{$+\mathrm{C} \mathrm{IV}$} \\
\hline [Ar IV] & 4711 & 0.004 & .001 & - & - \\
\hline \multicolumn{6}{|l|}{$+\mathrm{He} \mathrm{I}$} \\
\hline $\mathrm{H} \beta$ & 4861 & 1 & .1 & 1 & .1 \\
\hline He I & 4921 & 0.011 & .004 & 0.006 & .001 \\
\hline [O III] & 4959 & 1.7 & .1 & 1.41 & .08 \\
\hline [O III] & 5007 & 5.1 & .3 & 4.2 & .2 \\
\hline$[\mathrm{N} \mathrm{I}]$ & 5198 & - & - & 0.005 & .001 \\
\hline [Cl III] & 5517 & 0.001 & .000 & 0.023 & .001 \\
\hline [Cl III] & 5537 & 0.003 & .000 & 0.02 & .01 \\
\hline $\mathrm{HeI}$ & 5875 & 0.063 & .001 & 0.120 & .004 \\
\hline$[\mathrm{O} \mathrm{I}]$ & 6300 & 0.011 & .007 & 0.029 & .009 \\
\hline [S III $]$ & 6312 & 0.006 & .004 & 0.012 & .004 \\
\hline$[\mathrm{O} I]$ & 6363 & 0.004 & .001 & 0.009 & .002 \\
\hline$\left[\begin{array}{ll}\mathrm{N} & \mathrm{II}\end{array}\right]$ & 6548 & 0.006 & .004 & - & - \\
\hline $\mathrm{H} \alpha$ & 6562 & 1.50 & .07 & 2.859 & .400 \\
\hline$\left[\begin{array}{ll}\mathrm{N} & \mathrm{II}\end{array}\right]$ & 6583 & 0.031 & .009 & 0.073 & .008 \\
\hline He I & 6678 & 0.016 & .003 & 0.030 & .005 \\
\hline$[\mathrm{S}$ II $]$ & 6716 & 0.05 & .01 & 0.11 & .03 \\
\hline$[\mathrm{S}$ II $]$ & 6730 & 0.03 & .01 & 0.07 & .02 \\
\hline$C_{\beta}$ & & 1.9 & & 1.4 & \\
\hline$E_{\mathrm{v}}(\mathrm{H} \beta)$ & & 234 & & 165 & \\
\hline$\sigma(l)_{4363}$ & & 17 & & 15 & \\
\hline $\log F(\mathrm{H} \beta)$ & & -12.16 & & -12.41 & \\
\hline
\end{tabular}



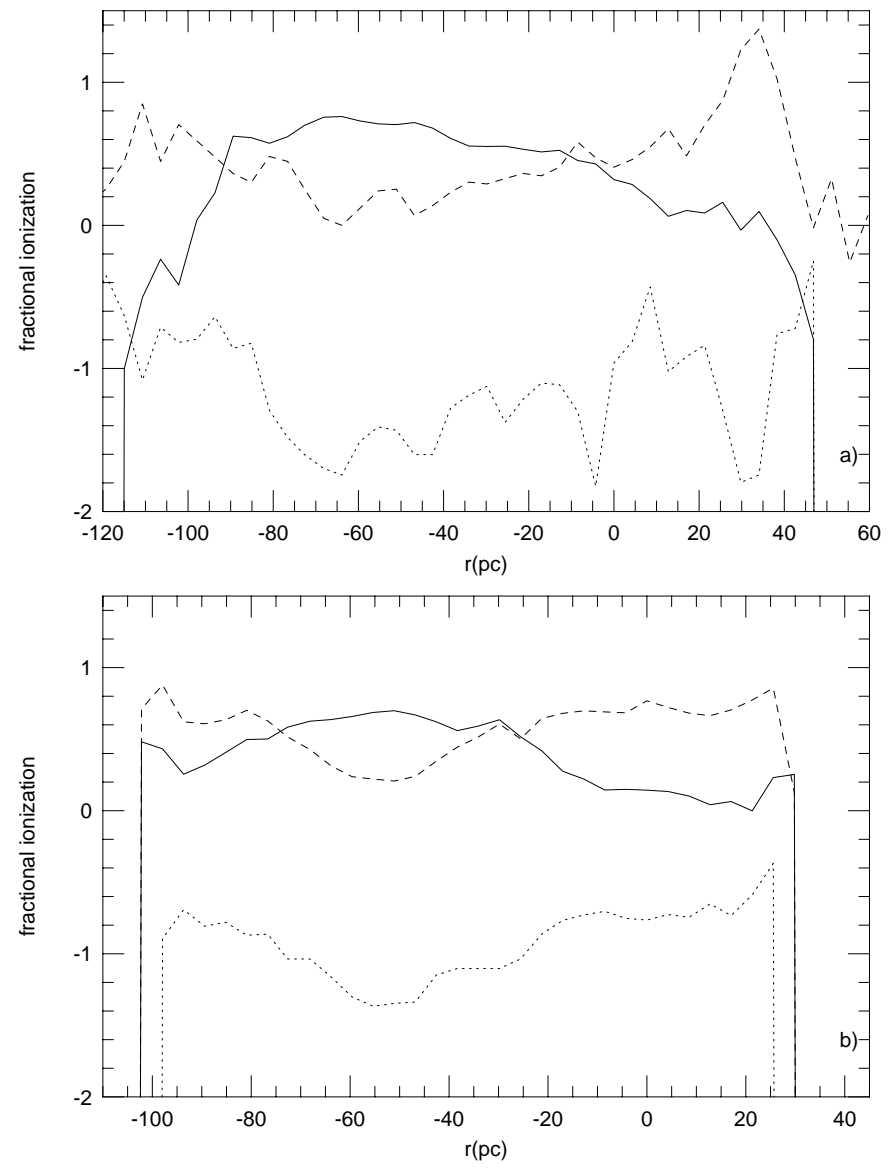

Fig. 6. The fractional ionization of Hubble $\mathrm{V}$ a) and $\mathrm{X} \mathbf{b}$ ) defined as; $[\mathrm{OIII}] / \mathrm{H} \beta(-),[\mathrm{OII}] / \mathrm{H} \beta(--)$ and $[\mathrm{NII}] / \mathrm{H} \beta$ $(\cdots)$

absolute values change. The physical size of each region is measured from the geometrical center of the nebula determined from the $\mathrm{H} \alpha$ emission in the spectra. The western part corresponds to positive values. A region between -80 and $-20 \mathrm{pc}$ and -80 and $-30 \mathrm{pc}$ could be defined for Hubble $\mathrm{V}$ and Hubble X, respectively, where $\log (\sigma(l))$ is higher than 0.9 (based on the intensity of the $\mathrm{H} \alpha$ emission line) or 0.5 (based on the intensity of [OIII] $\lambda 4363 \AA$ ), where the signal-to-noise ratio was considered acceptably high for a meaningful analysis (Rola \& Pelat 1994).

Therefore, the study of possible spatial variations in the physical properties of Hubble $\mathrm{V}$ and Hubble $\mathrm{X}$ is considered limited by the signal-to-noise ratio obtained and only applies to the parts of each H II region where this ratio is sufficiently high. It should be pointed out that in the case of Hubble V (Fig. 5a) some limitations exist. From the figure it is evident that the signal-to-noise ratio of the $[\mathrm{OIII}] \lambda 4363 \AA$ line shows a steep gradient close to $-80 \mathrm{pc}$. Also, this line is practically undetected close to $-35 \mathrm{pc}$ and closer to the geometrical center the signal-tonoise ratio is generally low. The situation for Hubble $\mathrm{X}$ (Fig. 5b) is more favourable.
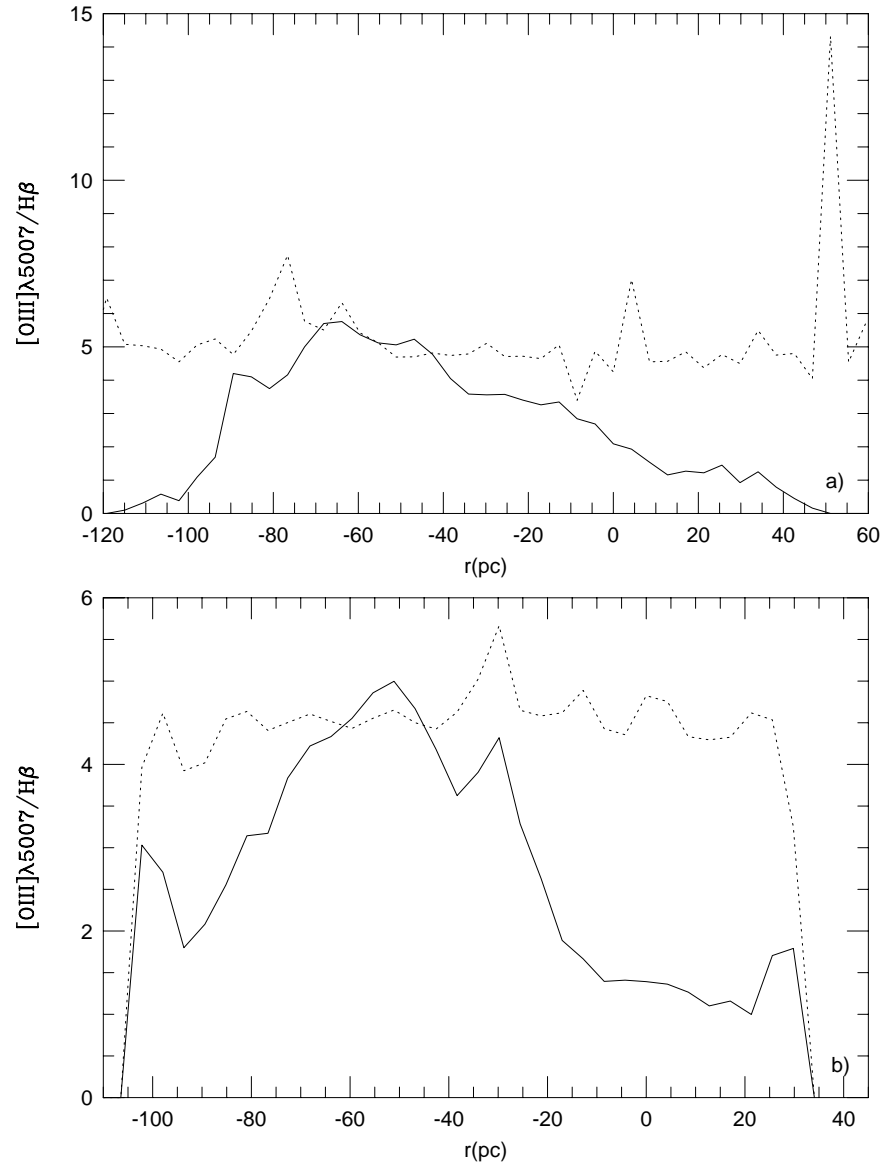

Fig. 7. The excitation parameter, defined as [OIII $] \lambda 5007 / \mathrm{H} \beta$ $(-)$, of Hubble $\mathrm{V} \mathbf{a}$ ) and Hubble $\mathrm{X} \mathbf{b}$ ). The strong western peak evident in Hubble $\mathrm{V}$ is probably an effect of the low signalto-noise ratio. The non reddening-corrected $\mathrm{H} \alpha / \mathrm{H} \beta$ ratio is also presented $(\cdots)$

\subsubsection{The ionization structure}

The ionization structure of Hubble $\mathrm{V}$ and Hubble $\mathrm{X}$ was studied. The low and high ionization zones are plotted in Figs. $6 \mathrm{a}$ and b. They correspond to the regions of the maximum intensity of $\mathrm{O}^{+}$and $\mathrm{O}^{++}$, respectively. A general definition of a high ionization region could be those geometrical locations where the ratio $\mathrm{O}^{++} / \mathrm{O}^{+}$is higher than unity. The high ionization region is limited to between -90 and $-10 \mathrm{pc}$ in Hubble $\mathrm{V}$ and to -70 and $-20 \mathrm{pc}$ in Hubble $\mathrm{X}$. In both $\mathrm{H}$ II regions, the high ionization region coincides with the peaks in the excitation parameter (see the next section). It is also evident from Figs. $6 \mathrm{a}$ and $\mathrm{b}$ that the $\mathrm{O}^{+}$zone coincides quite well with the $\mathrm{N}^{+}$zone. This gives strong support for the use of the ratio $N(\mathrm{O}) / N\left(\mathrm{O}^{+}\right)$as an ICF for nitrogen (see Sect. 5.2.2).

\subsubsection{The excitation structure}

The excitation parameter is defined as the ratio of the two emission lines $[\mathrm{OIII}] \lambda 5007 / \mathrm{H} \beta$. Peaks in the excitation parameter are seen at -65 and -55 pc in Hubble $\mathrm{V}$ and Hubble X (Figs. 7a and b), respectively. This could be an 
indication of the location of the $\mathrm{OB}$ association within the nebula, which is ionizing the region.

When comparing the two regions, it is evident that they show quite a high level of excitation, with Hubble $\mathrm{V}$ displaying somewhat higher absolute values across the face of the object. There could be two reasons for this: The most recent episode of star formation is younger in Hubble $\mathrm{V}$ or the ionizing stars are hotter, and therefore, more massive. A comparison of the equivalent width of $\mathrm{H} \beta, E_{\mathrm{v}}(\mathrm{H} \beta)$, supports the first possibility, provided this parameter traces the age of the region for a given IMF (Copetti et al. 1986). This is in agreement with the conclusion by Wilson (1992). The age of Hubble X is determined by the latter from colour-magnitude diagrams and suggests that it is 1.6 Myr older than the age of the last event of star formation in Hubble V. Also, the OB association in Hubble $\mathrm{V}$ contains four more massive stars than that of Hubble X.

The non reddening-corrected $\mathrm{H} \alpha / \mathrm{H} \beta$ ratio is also presented in Figs. 7a and b. The $\mathrm{H} \alpha / \mathrm{H} \beta$ ratios of both regions are rather high, values of 5 and 4.5 for Hubble $\mathrm{V}$ and Hubble X were derived, respectively. These numbers are close to those reported in the literature for these regions. Talent (1980) obtained non-corrected intensities of 5.4 and 4.5 for Hubble V and Hubble X, respectively. It should be emphasized again that these values are not corrected for galactic extinction (see Sect. 4.2).

The $[\mathrm{OIII}] /[\mathrm{OII}]$ ratio is also an indicator of excitation and has a maximum at $-65 \mathrm{pc}$ in the case of Hubble $\mathrm{V}$. The same number for Hubble $\mathrm{X}$ is $-50 \mathrm{pc}$. These positions coincide very well with the maxima of the excitation parameter and is expected since the $\mathrm{O}^{+}$-zone is largely temperature independent.

\subsubsection{Real abundance variations or local temperature fluctuations?}

It is well known that temperature fluctuations in the nebular gas could mimic variations in the abundance of chemical elements. Temperatures derived from the nebular continuum are distinctly lower than those derived from the collisionally-excited emission lines, which could be the cause of the different temperature measurements (Peimbert 1967). González-Delgado et al. (1994) studied the giant $\mathrm{H}$ II region NGC 2363 located in the irregular galaxy NGC 2366. They compared the average temperatures derived from the Paschen continuum emission and the [OIII] lines and found differences of at least $2000 \mathrm{~K}$ using the two methods. Liu \& Danziger (1993) derived the same result from a study of 14 planetary nebulae $(\mathrm{PNe})$. On the other hand, Liu et al. (1995) used only the Balmer discontinuity in a study of the Orion nebula and found that the temperature seems constant, close to $9000 \mathrm{~K}$, over a large portion of the nebula. Obviously, the situation is rather confusing and, in this analysis, insufficient spectral information was available, e.g. from the Balmer
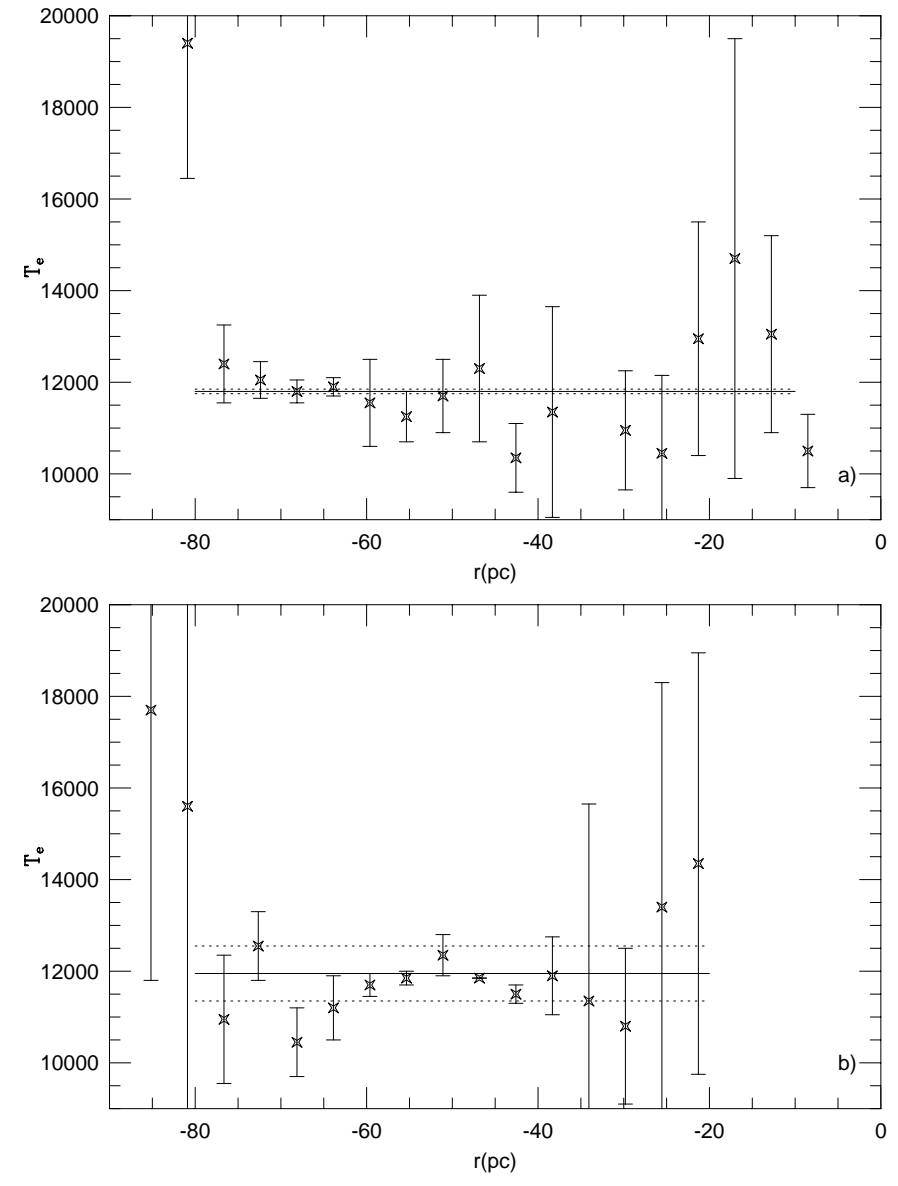

Fig. 8. The electron temperature, $T_{\mathrm{e}}$, over the face of Hubble $\mathrm{V}$ a) and $\mathrm{X} \mathrm{b}$ ). The horizontal lines (solid) correspond to the average value (solid) determined from the spatially-averaged spectra with a $1 \sigma$ deviation (dashed)

or Paschen discontinuities and no such comparison could be made.

\subsubsection{Electron temperatures and oxygen abundances}

Figures $8 \mathrm{a}$ and $\mathrm{b}$ show the distribution of the $T_{\mathrm{e}}$ over the face of Hubble $\mathrm{V}$ and Hubble $\mathrm{X}$, respectively, for which average values of $11500 \mathrm{~K}$ and $12000 \mathrm{~K}$ were derived. For the spatially-averaged spectra, the $T_{\mathrm{e}}$ are $11800 \pm 50 \mathrm{~K}$ and $11950 \pm 600 \mathrm{~K}$ for Hubble $\mathrm{V}$ and $\mathrm{X}$, respectively (see Table 5) and are marked in the figures with horizontal lines. The spatial distribution of oxygen, determined with the use of the temperature-sensitive method (Osterbrock 1989) over the face of Hubble $\mathrm{V}$ is presented in Fig. 9a. The same is given for Hubble $\mathrm{X}$ in Fig. 9b. As expected, the $T_{\mathrm{e}}$ and oxygen abundance are distributed in the same manner. It is evident that some variations in oxygen abundance are found. They could reflect real variations in oxygen abundance or small temperature fluctuations. An average value of 8.15 is obtained for both regions, which agrees well with the abundances obtained from the spatially-averaged spectra (Table 5). We note the very small uncertainties derived for the center of Hubble X. 

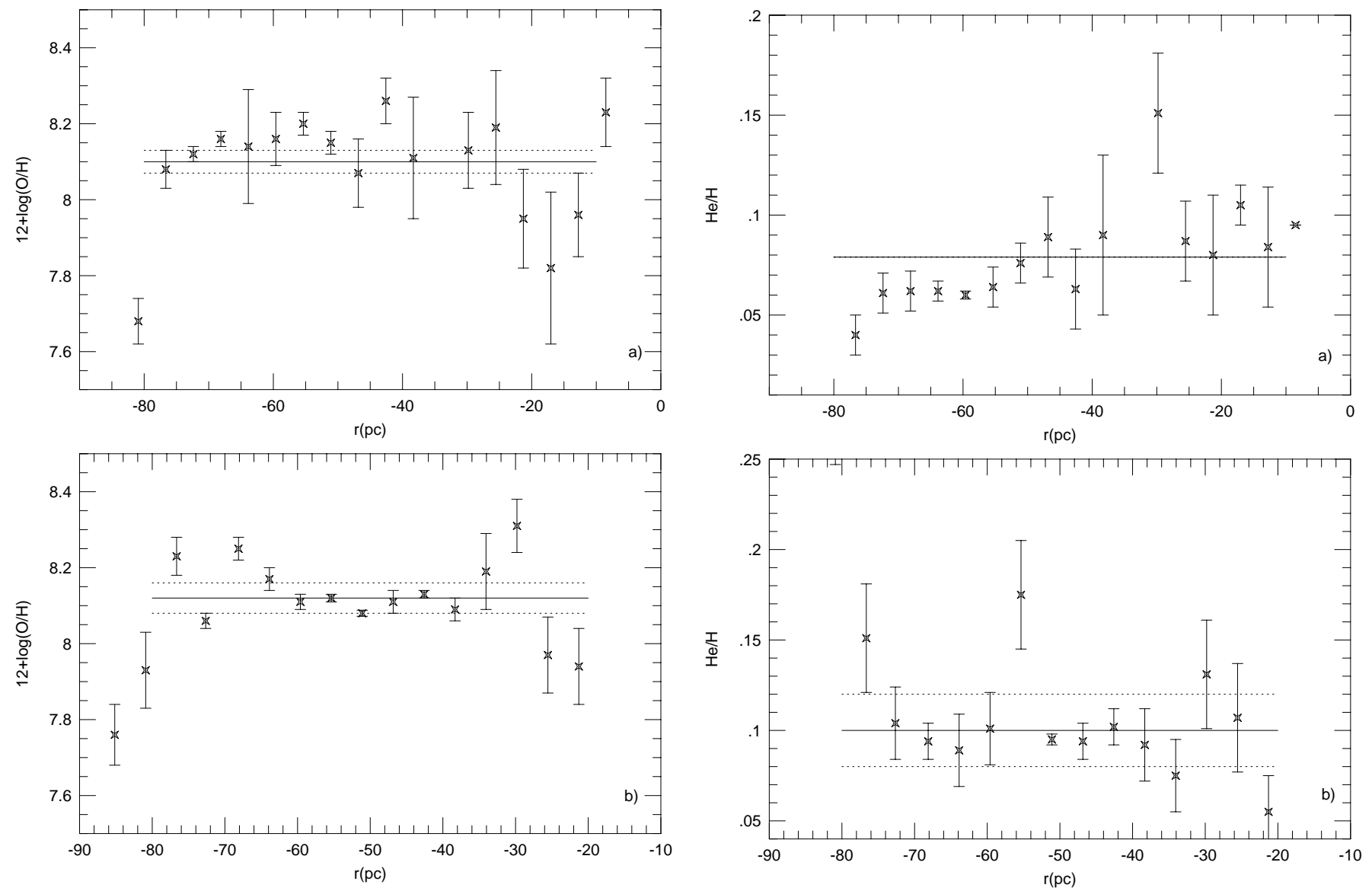

Fig. 9. The spatial distribution of $(\mathrm{O} / \mathrm{H})$ in Hubble $\mathrm{V}$ a) and Hubble $\mathrm{X} \mathbf{b}$ ). The region with sufficiently high signal-to-noise ratio is considered to be between -80 and $-20 \mathrm{pc}$ for Hubble $\mathrm{V}$ and between -80 and $-30 \mathrm{pc}$ for Hubble $\mathrm{X}$. The horizontal lines correspond to the average value (solid) determined from the spatially-averaged spectra with a $1 \sigma$ deviation (dashed)

\subsubsection{The spatial distribution of helium, nitrogen and neon}

In order to study metallicity gradients within extragalactic H II regions, the distribution of some other elements, such as helium and nitrogen, should be investigated. Many studies have been devoted to the abundances of these elements in galaxies with moderately low metallicity, e.g. Garnett (1989), Garnett (1990) and Izotov et al. (1997). Apart from oxygen, only a few studies have been performed on the variation and possible inhomogeneities of these elements within the HII regions (Kobulnicky \& Skillman 1996; Kobulnicky et al. 1997). Some other traditionally controversial topics, such as the origin of nitrogen or the abundance of primordial helium, have been analysed instead.

The emission lines used for a determination of helium abundances are normally HeI $\lambda 4471 \AA$, HeI $\lambda 5875 \AA$ and HeI $\lambda 6678 \AA$. Other HeI (and, if present, HeII) lines can also be used. Taking into consideration that some lines could be affected by fluorescence and underlying stellar absorption (Izotov et al. 1997), a weighted average value for the helium line intensity was obtained. Only two of

Fig. 10. The spatial distribution of $(\mathrm{He} / \mathrm{H})$, by number, in Hubble V a) and Hubble X b). The horizontal lines correspond to the average value (solid) determined from the spatiallyaveraged spectra with a $1 \sigma$ deviation (dashed)

the above mentioned lines were used for the abundance determination. The line He $\lambda 5875 \AA$ was excluded from the abundance determination due to its proximity to the NaI $\lambda \lambda 5890,5896 \AA$ lines. The latter can be of stellar as well as interstellar origin.

Figures 10a and b show the spatial distribution of helium, by number, over the face of both Hubble $\mathrm{V}$ and Hubble X. The situation for Hubble V (Fig. 10a) is somewhat confusing. The figure shows strong variations in the $(\mathrm{He} / \mathrm{H})$ ratio over the face of the object. In the eastern part, the values are rather close to those predicted by the hot Big Bang model. However, considering the low signalto-noise ratio in parts of the spectra of Hubble V, this behaviour is to be expected. The helium abundance within Hubble X (Fig. 10b), on the other hand, is constant at $(\mathrm{He} / \mathrm{H})=0.10$.

The distribution of nitrogen in both regions is presented in Figs. 11a and b. In the parts of the $\mathrm{H}$ II regions considered, the nitrogen abundance is more or less constant, close to $\log (\mathrm{N} / \mathrm{H})=-5.60$ and -5.50 for Hubble $\mathrm{V}$ and Hubble X, respectively. These values agree with those derived from the spatially-averaged spectra (Table 5). The derived numbers are rather typical for this class of objects (e.g. Izotov et al. 1997). Some variations could be 
Table 5. The spatially-averaged parameters derived for the $\mathrm{H}$ II regions Hubble $\mathrm{V}$ and Hubble $\mathrm{X}$. The bottom row refers to a determination of the oxygen abundance with the use of the semi-empirical method (McGaugh 1994)

\begin{tabular}{ccc}
\hline & Hubble $\mathrm{V}$ & Hubble X \\
\hline$T_{\mathrm{e}}\left(\mathrm{O}^{++}\right)$ & $11800( \pm 50) \mathrm{K}$ & $11950( \pm 600) \mathrm{K}$ \\
$T_{\text {ion }}$ & $48500( \pm 3900) \mathrm{K}$ & $46000( \pm 3100) \mathrm{K}$ \\
$12+\log (\mathrm{O} / \mathrm{H})$ & $8.10( \pm .03)$ & $8.12( \pm .04)$ \\
$\mathrm{ICF}(\mathrm{N})$ & $3.2( \pm .8)$ & $5.0( \pm .1)$ \\
$12+\log (\mathrm{N} / \mathrm{H})$ & $6.5( \pm .2)$ & $6.4( \pm .3)$ \\
$\log (\mathrm{N} / \mathrm{O})$ & $-1.58( \pm .04)$ & $-1.7( \pm .1)$ \\
$\mathrm{ICF}(\mathrm{Ne})$ & $1.4( \pm .2)$ & $1.2( \pm .3)$ \\
$12+\log (\mathrm{Ne} / \mathrm{H})$ & $7.3( \pm .1)$ & $7.4( \pm .3)$ \\
$\log (\mathrm{Ne} / \mathrm{O})$ & $-0.78( \pm .05)$ & $-0.8( \pm .2)$ \\
$\mathrm{He} / \mathrm{H}$ & $0.079( \pm .002)$ & $0.10( \pm .02)$ \\
$\mathrm{Y}$ & $0.240( \pm .005)$ & $0.289( \pm .007)$ \\
$12+\log (\mathrm{O} / \mathrm{H})_{\text {sem }}$ & 8.07 & 8.09 \\
\hline
\end{tabular}

argued for, especially the overabundance of nitrogen close to $-30 \mathrm{pc}$ in Hubble $\mathrm{V}$ (Fig. 11a). However, considering the uncertainties, no firm conclusions regarding the presence of fluctuations in nitrogen abundance in the region could be drawn, since this could be an effect of the low signal-to-noise ratio.

The spatial distribution of neon over the face of the two $\mathrm{H}$ II regions is presented in Figs. 12a and b. The lines used for the determination of the abundance of neon, [NeIII] $\lambda 3869 \AA$ and [NeIII] $\lambda 3970 \AA$, could be affected by metal-line blanketing. Due to the relatively low metallicity of these regions, this effect should not be too crucial for this analysis, even though some uncertainties are introduced. The average values obtained, $\log (\mathrm{Ne} / \mathrm{H})=-4.70$ and -4.60 for Hubble $\mathrm{V}$ and Hubble X, respectively, show good agreement with the spatially-averaged abundances (Table 5).

\subsection{The spatially-averaged abundances of Hubble $V$ and Hubble $X$}

As previously mentioned, a spectrum for each region was obtained by summing all the pixels where the line [OIII] $] 4363 \AA$ was detected. The total number of pixels for Hubble $\mathrm{V}$ and Hubble $\mathrm{X}$ corresponded to sizes of 75 and $67 \mathrm{pc}$, respectively. These two spatially-averaged spectra were corrected for extinction and absorption as described previously. Table 4 presents the intensities, normalized to $\mathrm{H} \beta$, and the total error estimate of each line, as described in Sect. 3. The extinction parameters, determined using $\mathrm{H} \gamma$ for Hubble $\mathrm{V}$ and $\mathrm{H} \alpha$ for Hubble $\mathrm{X}$, are also given. The equivalent width and the flux of $\mathrm{H} \beta$ as well as the signal-to-noise ratio measured in the vicinity of the line [OIII] $\lambda 4363 \AA$ are presented in the table as well.
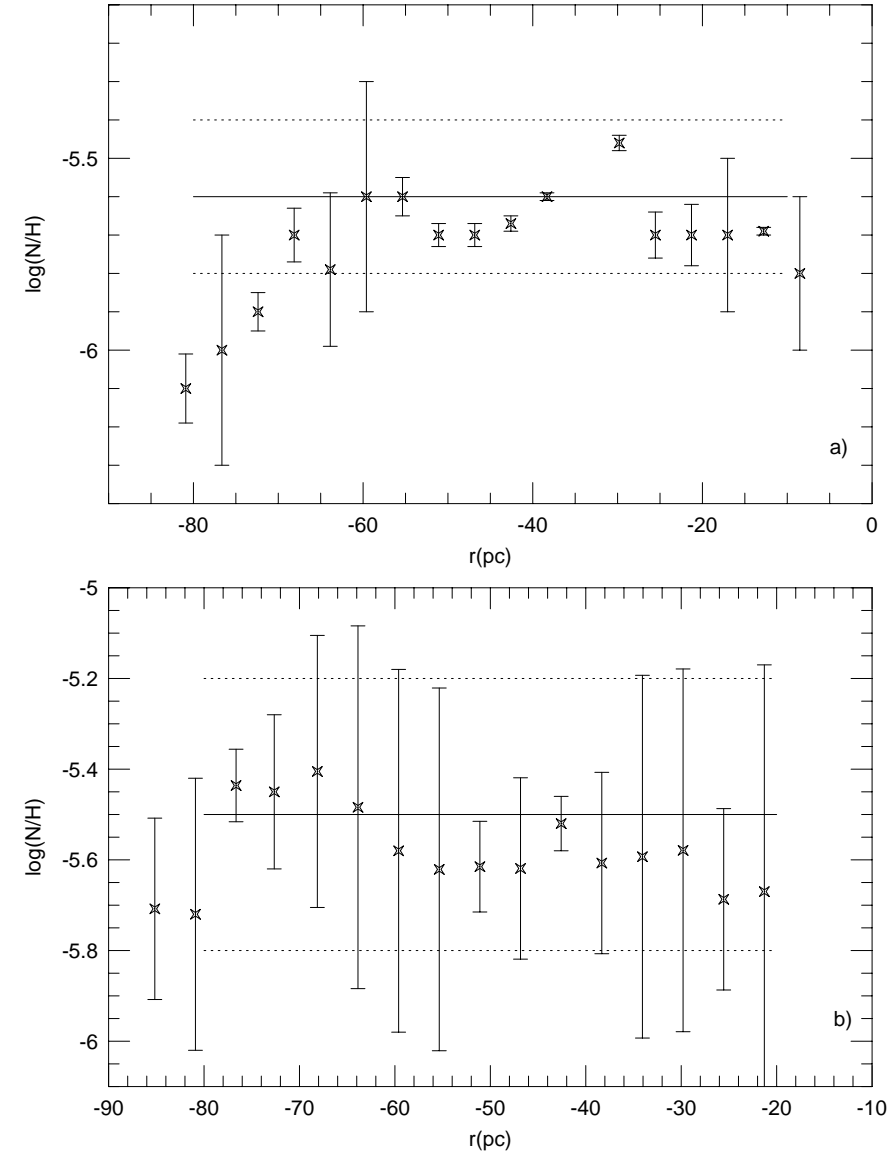

Fig. 11. The spatial distribution of $(\mathrm{N} / \mathrm{H})$ in Hubble $\mathrm{V}$ a) and Hubble $\mathrm{X} \mathbf{b}$ ). The horizontal lines correspond to the average value (solid) determined from the spatially-averaged spectra with a $1 \sigma$ deviation (dashed)

The spatially-averaged abundances of helium, nitrogen, neon and oxygen are presented in Table 5, as well as the corresponding ICFs. The abundance ratios $\log (\mathrm{N} / \mathrm{O})$ and $\log (\mathrm{Ne} / \mathrm{O})$ are also presented. Finally, the $T_{\mathrm{e}}$ and the temperature of the ionizing radiation, $T_{\text {ion }}$ (see Sect. 5.5), derived for the two regions are given, as well as the oxygen abundances obtained with the semiempirical method (McGaugh 1994).

The total helium abundance was obtained, as in the case of the $3 \mathrm{r}$ spectra, from the weighted sum of the helium abundance from the lines $\operatorname{He} \lambda 4771 \AA$ and $\operatorname{He} \lambda 6678 \AA$. No ICF was used for the reasons discussed in Sect. 5.2.1. Both $(\mathrm{He} / \mathrm{H})$ and $\mathrm{Y}$ (the mass fraction of helium) are compiled in Table 5.

The ratio $\log (\mathrm{N} / \mathrm{O})$ has been determined for both regions (Table 5). From a comparison with a sample of dI (Garnett 1990) and blue compact galaxies (BCG) (Izotov \& Thuan 1999) it can be concluded that Hubble X has a value slightly lower than the average derived from those investigations, which is $\log (\mathrm{N} / \mathrm{O})=-1.5$.

The ratio $\log (\mathrm{Ne} / \mathrm{O})$ is -0.78 and -0.8 for Hubble $\mathrm{V}$ and Hubble X, respectively. A comparison of the sample of dI by McCall et al. (1985) and BCG (Izotov \& Thuan 1999) was made. It can be concluded that the spatiallyaveraged ratio is normal for this type of object. One should 

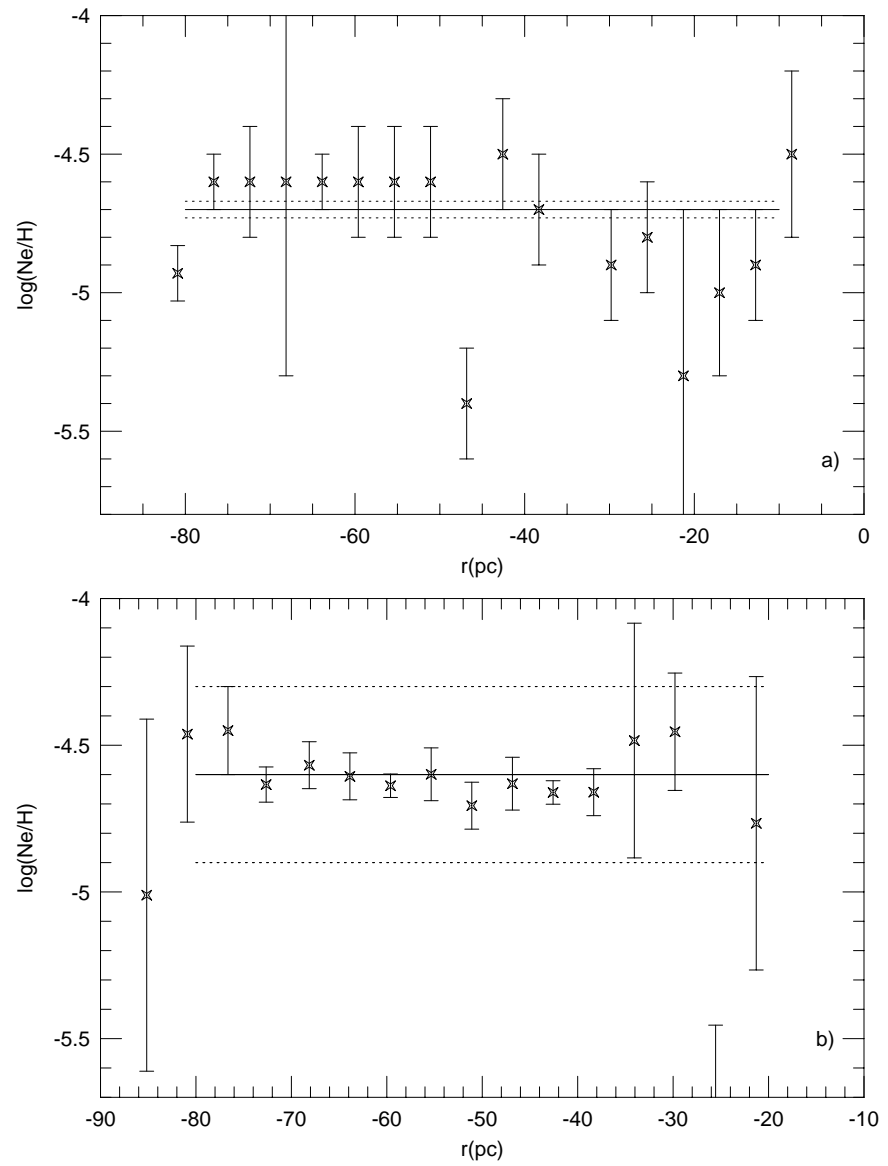

Fig. 12. The spatial distribution of $(\mathrm{Ne} / \mathrm{H})$ in Hubble $\mathrm{V}$ a) and Hubble $\mathrm{X} \mathbf{b}$ ). The horizontal lines correspond to the average value (solid) determined from the spatially-averaged spectra with a $1 \sigma$ deviation (dashed)

also note the small difference in the total neon abundance between the two regions which is in good agreement with the results from the spatially-averaged oxygen abundance.

A number of studies focused on NGC 6822 was selected for comparison, such as Peimbert \& Spinrad (1970), Lequeux et al. (1979), Talent (1980), Pagel et al. (1980), and Skillman et al. (1989). The extinction coefficients and the elemental abundances from those investigations are presented in Table 6 . The oxygen abundance is reported in all the studies selected.

From an inspection of Tables 5 and 6 it is clear that the abundances obtained in this study agree well with those presented by Pagel et al. (1980). It should be pointed out that despite the relatively high extinction found for both regions, quite different from the one obtained by Pagel et al. (1980), the $T_{\mathrm{e}}$ 's and oxygen abundances for both H II regions are similar in both investigations. This is also true for $\log (\mathrm{N} / \mathrm{O})$ of Hubble $\mathrm{X}$. The differences obtained in the extinction values could be due to a different positioning of the slit within the HiI regions.

\subsection{The temperature of the ionizing radiation}

In order to more clearly understand the origin of some features observed in the spectra of Hubble $\mathrm{V}$ and Hubble
Table 6. Elemental abundances of Hubble V (HV) and Hubble $\mathrm{X}$ (HX) from previous investigations found in the literature (determined with the use of the [OIII] lines). The marking $(*)$ refers to ionic abundance ratios. References: 1) Peimbert \& Spinrad (1970); 2) Lequeux et al. (1979); 3) Pagel et al. (1980); 4) Talent (1980); 5) Skillman et al. (1989)

\begin{tabular}{ccccccc}
\hline & & 1 & 2 & 3 & 4 & 5 \\
\hline$C_{\beta}$ & HV & 0.32 & & 1.04 & & \\
& HX & & 0.75 & & & \\
$T_{\mathrm{e}}\left(\mathrm{O}^{++}\right)$ & $\mathrm{HV}$ & 12180 & 11510 & 11000 & 11410 & 11450 \\
& $\mathrm{HX}$ & & 10900 & 11500 & 10920 & \\
$12+\log (\mathrm{O} / \mathrm{H})$ & $\mathrm{HV}$ & 8.09 & 8.20 & 8.19 & 8.28 & 8.22 \\
& $\mathrm{HX}$ & & 8.27 & 8.09 & 8.31 & \\
$\log (\mathrm{N} / \mathrm{O})$ & $\mathrm{HV}$ & & -1.77 & -1.69 & & $-1.7^{*}$ \\
& $\mathrm{HX}$ & & & -1.74 & & \\
$\log (\mathrm{Ne} / \mathrm{O})$ & $\mathrm{HV}$ & & -0.66 & -0.85 & & \\
& $\mathrm{HX}$ & & -0.66 & -0.75 & & \\
$N(\mathrm{He}) / N(\mathrm{H})$ & $\mathrm{HV}$ & 0.094 & 0.081 & & & \\
& HX & & 0.084 & & & \\
\hline
\end{tabular}

X, e.g. the state of ionization, the $T_{\text {ion }}$ was derived. This was performed using a model of spectral evolution of Olofsson (1997). The $T_{\text {ion }}$ was determined by comparing the spectral energy distribution shortwards of the Lyman limit at a given age of a single-burst stellar population and metallicity with model stellar atmosphere spectra of Kurucz (1992) and Howarth \& Lynas-Gray (1989) at the corresponding metallicity in the temperature range $33000-55000 \mathrm{~K}$ and $\log g=4.0-5.0$. This was accomplished using a method of least square summation. The input to the model is the empirical oxygen abundance, the line ratios $R_{23}$ and $R_{33}$, defined as $R_{23}=([\mathrm{OII}] \lambda 3727+[\mathrm{OIII}] \lambda \lambda 4959,5007) / \mathrm{H} \beta$ and $R_{33}=$ $[\mathrm{OIII}] \lambda 4363 /[\mathrm{OIII}] \lambda \lambda(4959+5007)$, as well as the number of Lyman continuum photons, derived from the flux in the $\mathrm{H} \beta$ emission line of the two regions. Weighted average $T_{\text {ion's }}$ are shown in Table 5 , where those obtained from the $R_{23}$ ratio are given a higher weight due to the uncertainties in the measurements of the [OIII] $\lambda 4363 \AA$ line, which will strongly affect the $R_{33}$ ratio. As evident, the ionization temperature is rather high $\left(T_{\text {ion }}=48500\right.$ and $46000 \mathrm{~K}$ for Hubble V and Hubble X, respectively). This could explain the presence of emission lines such as $\operatorname{HeI} \lambda 4026 \AA$ and $[\mathrm{SII}] \lambda \lambda 4068+4076 \AA$ since these elements and ionization stages have approximately equal ionization potentials. The nebular [ArIV] line is present in the spectra which is not the case for the nebular HeII $\lambda 4685 \AA$ line. This is most likely due to the higher ionization potential of the HeII line.

A comparison of a sample of BCG where HeII was detected (Campbell et al. 1986) was made in order to determine how the $T_{\text {ion }}$ is linked to the appearance of HeII lines. The $T_{\mathrm{e}}$ 's were derived from Campbell et al. (1986) and the $T_{\text {ion's }}$ 'sere taken from Cerviño \& Mas-Hesse (1994). 
For the six galaxies where both temperatures were available, all except one (Tol 1004-296s), had $T_{\mathrm{e}}$ 's higher than $13000 \mathrm{~K}$ but the $T_{\text {ion }}$ 's proved to be very different ranging from $38900 \mathrm{~K}$ (Tol 1004-296s) to greater than $55000 \mathrm{~K}$ (Tol 1214-277). This indicates that the presence of HeII in an $\mathrm{HII}$ region is not necessarily related to a higher $T_{\text {ion }}$, and that there should be another parameter involved, e.g. the metallicity (Cerviño \& Mas-Hesse 1994; Olofsson 1997). In support of this is the fact that of the metallicity of the six galaxies in Cerviño \& Mas-Hesse (1994), all except one, Tol 1004-296s, have $12+\log (\mathrm{O} / \mathrm{H}) \leq 8$.

\subsection{A contribution from other sources of ionization?}

The major source of ionization in this type of object is photoionization by hot stars. However, a contribution to the total ionization by other sources, such as PN ejection, shock-heating from SN ejecta and non-thermal radiation, cannot, a priori, be ruled out. The intensity of some emission lines are strongly enhanced in the presence of other sources of ionization. The emission lines $[\mathrm{OI}] \lambda \lambda 6300$, $6363 \AA$ and $[\mathrm{ClIII}] \lambda \lambda 5517,5537 \AA$, are clearly present in the spectra of Hubble $\mathrm{V}$ and Hubble X. The [OI] lines, especially, indicate the presence of shock-heating or nonthermal emission. The latter source can be ruled out due to the absence of highly ionized species, such as FeX, or broad emission lines, which are typical of active galaxies.

Considering the uncertainties associated with the weak [OI] lines, their intensities measured in the HII regions studied here could be explained by pure photoionization (Stasińska 1990) and therefore no correction for shockheating to the line intensities was considered necessary.

The [ClIII] lines are mainly reported in studies of PNe (e.g. Saurer 1998) but also in some BCG, as evident in the sample of Izotov et al. (1997). A possibility is that these lines appear because of the high $T_{\text {ion }}$ in the regions. The ionization potential of [CIIII] is about $40 \mathrm{eV}$, which is smaller than that of He II $(54.4 \mathrm{eV})$. In this case, the lines could be ionized if the radiation is hard enough and is observable only when the data is of sufficiently high quality due to their low abundances.

In order to check whether PNe contribute to the total emission, diagnostic diagrams of Baldwin et al. (1981) were obtained for all the spectra where the lines were present. The diagrams which included the emission line ratios of $[\mathrm{OII}] \lambda 3727 \AA$, [OIII $] \lambda 5007 \AA$, [OI $] \lambda 6300 \AA$, $[\mathrm{NII}] \lambda 6583 \AA, \mathrm{H} \alpha$ and $\mathrm{H} \beta$ were used. It was obvious that, in all diagnostic diagrams, the observed line ratios were located within the position of ordinary H II regions ionized by hot stars. The same is true for the spectra where the [CIIII] line were detected. Even if the line ratios correspond to $\mathrm{H}$ II regions, the $\mathrm{PNe}$ could be positioned behind these, considering the weakness of the [CIIII] lines. Thus, the origin of the [CIIII] lines could be nebular or due to $\mathrm{PNe}$, but the relative amounts are difficult to assess. A comparison with photoionization models does not give a definitive answer due to uncertainties in the modeling as well as in the measurements.
Whatever the origin of chlorine, the abundance of [CIIII] can be estimated. These lines are not included in the software used for the abundance determination, but assuming that the $T_{\mathrm{e}}$ is roughly the same as that for the [OIII] region (Izotov et al. 1997), the expression for the nebular $P^{3}$ transitions from Aller (1984) could be used. The expression is

$$
\frac{N(\mathrm{Xi})}{N\left(\mathrm{H}^{+}\right)}=a L_{j}(x, t) \sqrt{t} E_{4,2}^{\mathrm{o}} 10^{d / t} \frac{I(\lambda)}{I\left(\mathrm{H}_{\beta}\right)}
$$

where $t=10^{-4} / T_{\mathrm{e}}$ is the diagnostic temperature parameter, $a, d$ and $E_{4,2}^{\mathrm{o}}$ are constants for each $t$ and $L_{j}(x, t)$ is a tabulated function which depends on the temperature, the density and the atomic transition. The numbers obtained are $N\left(\mathrm{Cl}^{++}\right) / N\left(\mathrm{H}^{+}\right)=0.83 \pm 0.210^{-8}$ and $8.55 \pm 210^{-8}$ for Hubble V and Hubble X, respectively. One could speculate whether the higher abundance of $\mathrm{Cl}^{++}$in Hubble $\mathrm{X}$ could have implications for the star formation history, e.g. the shape of the IMF, since PN ejection is restricted to stars of low mass. A simple explanation could be an excess of $\mathrm{PNe}$ in Hubble $\mathrm{X}$, if a harder radiation field in this region is ruled out. However, considering the uncertainties involved, in combination with the fact that only singly-ionized chlorine is observed, no conclusions regarding the relative number of $\mathrm{PNe}$ between the two regions can be drawn.

The abundances obtained can be compared to those of a sample of BCG (Izotov et al. 1997). The average value of $\mathrm{Cl}^{++}$for the 11 galaxies where [ClIII] is detected is $2.6510^{-8}$, which is an intermediate value between those for Hubble $\mathrm{V}$ and Hubble $\mathrm{X}$. Also, the ratio $\log \left(\mathrm{Cl}^{++} / \mathrm{O}^{++}\right)$can be determined. Values of -4.01 and -3.10 are obtained for Hubble $\mathrm{V}$ and Hubble $\mathrm{X}$, respectively. No direct comparison could be made with the results of the sample of Izotov et al. because they used the atomic abundances of chlorine but without giving any details regarding the explicit form of the ICF used.

\subsection{Small and large scale mixing of chemical elements}

\subsubsection{Small scale variations}

There are some gaseous mixing processes which act on scales of a few tens of parsecs. Local enrichment of gas ejected at SN explosions may be the most important, but also turbulent diffusion and fluid instabilities could have an effect on timescales of $10^{7}-10^{8}$ yr (Roy \& Kunth 1995) for $\mathrm{H}$ II regions of sizes of the order of $1 \mathrm{kpc}$. The regions studied in this analysis are about a factor of two smaller, which suggests that the timescales involved should be shorter by a factor of about ten. Even if turbulent diffusion and fluid instabilities are considered more efficient in large disk galaxies they cannot be completely ruled out in the case of dwarf galaxies.

The most remarkable characteristic of the oxygen distribution in Hubble V (Fig. 9a) is a deviation from the average value at $-43 \mathrm{pc}$ of 0.16 dex from the spatiallyaveraged value. A similar behaviour is noticed in Hubble $\mathrm{X}$ 

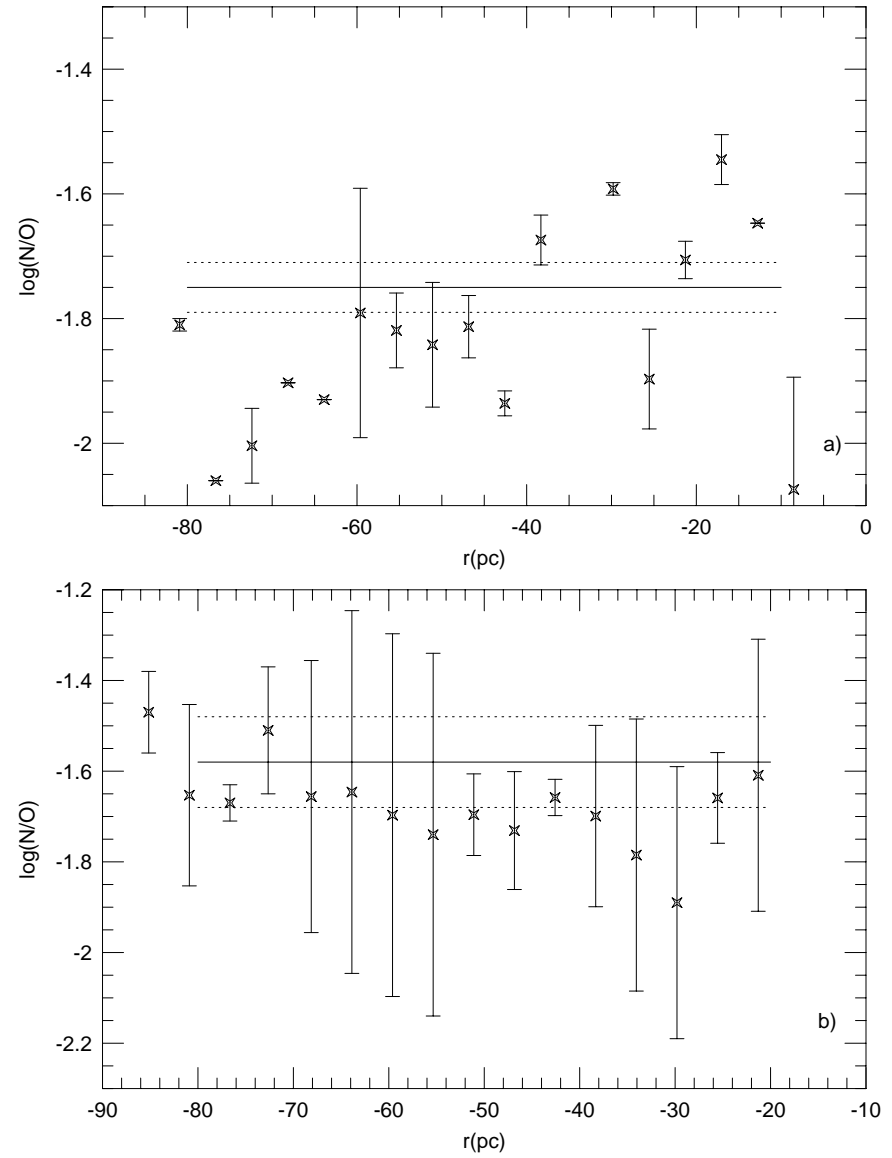

Fig. 13. The spatial distribution of $\log (\mathrm{N} / \mathrm{O})$ in Hubble $\mathrm{V}$ a) and Hubble $\mathrm{X} \mathbf{b})$. The horizontal lines correspond to the average value (solid) determined from the spatially-averaged spectra with a $1 \sigma$ deviation (dashed)

(Fig. 9b) at -68 pc of 0.13 dex from the spatially-averaged value. Considering the relatively small uncertainties measured at both locations, which included all the possible sources of uncertainty affecting the determination of the chemical abundances, the overabundances could not be ascribed to uncertainties in the oxygen abundance. This could represent real local overabundances of oxygen or could be an effect of local temperature fluctuations. If local enrichment from massive stars is the cause of the increase of the oxygen abundance, neon should show a similar behaviour, due to the fact that both elements are, mainly, the result of type II SN explosions with stars more massive than $\sim 10 M_{\odot}$ as progenitors. This is, however, difficult to assess, due to the high uncertainties involved, especially in the case of Hubble V.

As previously mentioned, an overabundance of nitrogen is detected at $-30 \mathrm{pc}$ in Hubble V (Fig. 11a). Also, at this location, one sees a variation in the helium abundance (Fig. 10a). The differences with respect to the average values are 0.15 dex in the nitrogen abundance and 0.25 dex in the helium abundance. This location also shows a difference in the $\log (\mathrm{N} / \mathrm{O})$ ratio with respect to the spatially-averaged value, evident in Fig. 13a. The distribution of $\log (\mathrm{N} / \mathrm{O})$ resembles that of $\log (\mathrm{N} / \mathrm{H})$. At the most western part, the large scatter is probably due to
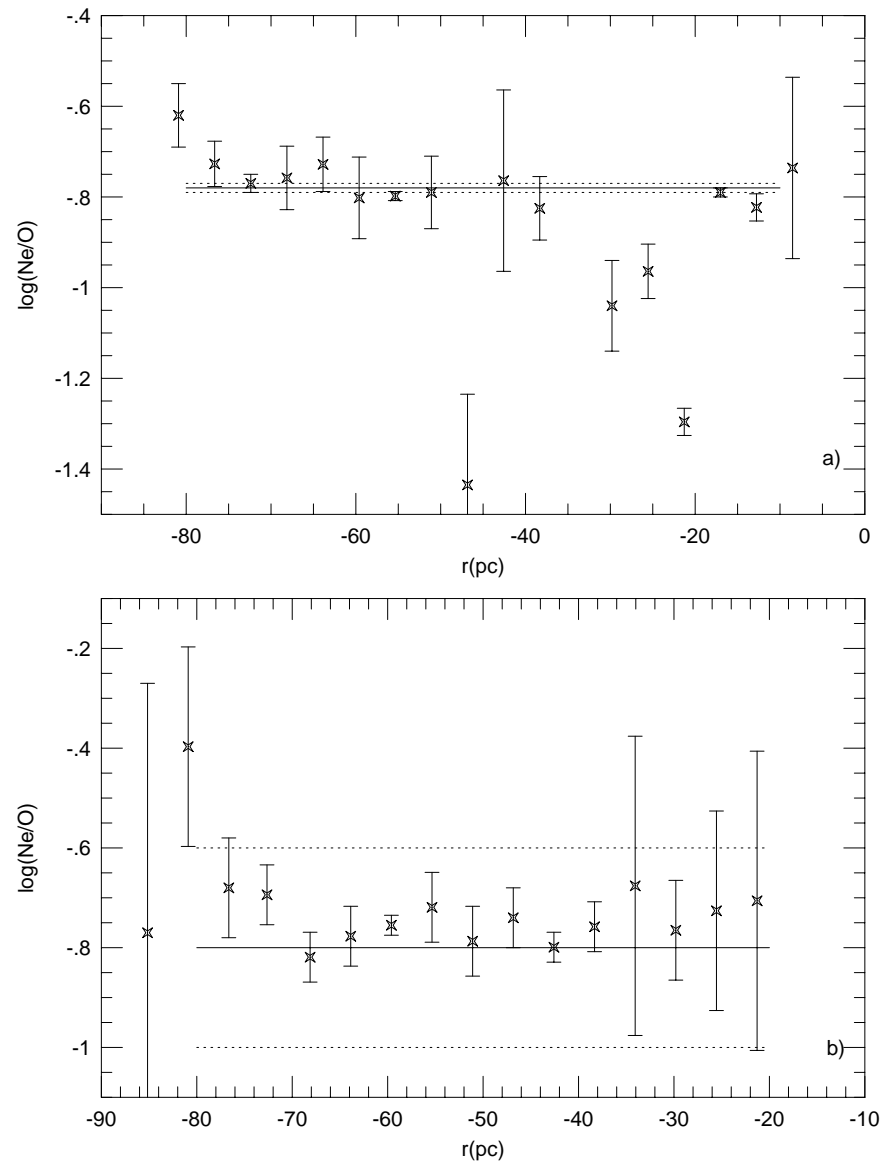

Fig. 14. The spatial distribution of $\log (\mathrm{Ne} / \mathrm{O})$ in Hubble $\mathrm{V}$ a) and Hubble $\mathrm{X} \mathbf{b}$ ). The horizontal lines correspond to the average value (solid) determined from the spatially-averaged spectra with a $1 \sigma$ deviation (dashed)

the low abundance of oxygen. The high value of the oxygen abundance at $-45 \mathrm{pc}$ corresponds to a low value in the $\log (\mathrm{N} / \mathrm{O})$ ratio, which indicates a real overabundances in oxygen. Figure $13 \mathrm{~b}$ shows the $\log (\mathrm{N} / \mathrm{O})$ ratio for Hubble $\mathrm{X}$ and it is clear that no major deviations from the average value are found across the $\mathrm{H}$ II region.

A possible explanation for the overabundances in helium and nitrogen could involve Wolf-Rayet stars. It is well known that winds from these stars could pollute the ISM (e.g. Maeder 1991). As previously mentioned, features typical of this type of star are absent from the spectra of Hubble $\mathrm{V}$ and $\mathrm{X}$. Considering the typical temperature of the winds expelled from the stars, the cooling time needed to observe the elements in the optical region is longer $\left(10^{7}\right.$ years) than the typical lifetime of the spectral features, assuming a short episode of star formation (which is of the order of $10^{5}$ years). The lack of helium or nitrogen overabundances in the so-called Wolf-Rayet galaxies (Kobulnicky et al. 1997) is suggestive of such a situation.

Figures $14 \mathrm{a}$ and $\mathrm{b}$ show the spatial distribution of $\log (\mathrm{Ne} / \mathrm{O})$ over the face of Hubble $\mathrm{V}$ and $\mathrm{X}$, respectively. A deviation from a constant value could be interpreted as a variation in the distribution of oxygen or neon due to mechanisms other than gas ejection from massive stars. 
The results show that the $\log (\mathrm{Ne} / \mathrm{O})$ ratio is largely constant, especially in Hubble X, which indicates a similar origin for both elements. The local underabundance in the $\log (\mathrm{Ne} / \mathrm{O})$ ratios at $-47 \mathrm{pc}$ and $-22 \mathrm{pc}$ in Hubble $\mathrm{V}$ could indicate a real depletion of neon, with respect to oxygen. One can also note that these locations are not spatially coincident with the higher-than-average locations in oxygen abundance (Fig. 9a). This could rule out a local enrichment from massive stars.

\subsubsection{Large scale mixing of chemical elements: A comparison between Hubble $\mathrm{V}$ and Hubble $\mathrm{X}$}

Another objective of this paper is to study possible variations in the chemical content, comparing $\mathrm{H}$ II regions in $\mathrm{dI}$. A more detailed discussion will be presented in a second paper (Hidalgo-Gámez et al. 2001).

Different mechanisms at different length- and timescales are responsible for a chemically well-mixed medium (Roy \& Kunth 1995). Epicyclic mixing for rotating disk galaxies or the presence of bars and superbubble expansion, among other phenomena, would erase any abundance variations on timescales less than $10^{9} \mathrm{yr}$. In the case of low-mass dwarf galaxies, some of the above mentioned mechanisms are less efficient or do not operate at all and the mixing of the elements in the ISM would be severely delayed. Other processes, such as selective loss of chemical elements through galactic winds and the low efficiency of mixing in a hot interstellar medium would make the variations larger in dwarf galaxies with long dormant phases between star-forming events.

The derived parameters for the two H II regions are presented in Table 5. It is clear that the oxygen abundance in both regions is about the same, within the uncertainties. For nitrogen and neon no major differences between the regions are found. The situation for helium is different. As evident from Table 5 , the $(\mathrm{He} / \mathrm{H})$ abundance ratio in Hubble $\mathrm{V}$ is about $2 / 3$ that of Hubble $\mathrm{X}$. This difference is certainly larger than the uncertainties in the measurements and should be considered real. The reason for the different values derived is unclear, especially in light of the fact that the other elements are similar if the two H II regions are compared. Uncertainties in the ICF can be ruled out since no HeII lines are observed in either spectra which implicate that the ICF should be close to one.

One possible explanation for the similarity in internal physical properties is probably the proximity between Hubble V and X, about 320 pc. Both H II regions are located in the same part of the galaxy, the northern edge of the bar. An argument favouring the efficient mixing of chemical elements between these H II regions is the existency of a bar which will flatten the abundance gradient, not only along, but also across the bar. Moreover, from the properties of the $\mathrm{OB}$ associations found in NGC 6822 (Wilson 1992) the star formation processes are similar. Also, the star formation history of both regions could be very similar. Israel et al. (1996) derived a recent star formation rate of $0.02 M_{\odot} \mathrm{yr}^{-1} \mathrm{kpc}^{-2}$ from infrared data for both regions. With this in mind, and considering the proximity of the two regions, it could be concluded that the mixing of the elements in the medium in the vicinity of Hubble $\mathrm{V}$ and Hubble $\mathrm{X}$ has been very efficient and the abundances of chemical elements are very similar, with differences of the same order as the error estimates.

Apart from possible inhomogeneities from previous events of star formation in the region, ongoing events could pollute the ISM of each region. Comparing the ages, 6.6 and 8.2 Myr, respectively, and the time the elements ejected from SNe need in order to reach the temperature of the ISM (typically of the order of $50 \mathrm{Myr}$ ), it is clear that those elements are too hot to be observed in the optical region. The possible chemical inhomogeneities detected in the optical region will be due to poor mixing of the elements from previous star-forming events.

\section{Conclusions}

A detailed study of the distribution of helium, nitrogen, oxygen and neon within the two major H II regions, Hubble $\mathrm{V}$ and Hubble X, in the dI galaxy NGC 6822 was made. Due to the high quality of the data, elemental abundances in regions of approximately $4.3 \mathrm{pc}$ were derived. The study of local enrichment in galaxies at small scales has been made previously, mainly for oxygen. Studies of variations in the helium, nitrogen and oxygen abundances in this type of object on scales as small as 4 pc have not previously been reported in the literature.

Indications of variations in the chemical abundances of oxygen, and possibly nitrogen, were found within the two H II regions. This could be real local chemical overabundances or could simply reflect local temperature fluctuations. Also, the extinction of Hubble $\mathrm{V}$ and Hubble $\mathrm{X}$ was studied and indicated an origin external to the $\mathrm{H}$ II regions. The physical properties of Hubble $\mathrm{V}$ and Hubble $\mathrm{X}$ are similar. This is related to the timing of the last episode of star formation, the star formation rates and the chemical abundances. This suggests that, due to the proximity of the two HiI regions, they could have influenced each other dynamically.

Acknowledgements. Dr. N. Bergvall, Dr. P. Leisy, Sr. V. Ortega, Mr. A. Pharasyn, Sr. Roman, Sr. Santillán, Dr. Sánchez-Salcedo and Dr. G. Östlin are acknowledged for their different contributions to this work. This paper has benefited much from the comments of Prof. J.-R. Roy. A.M.H.G was partially supported by NOTSA and the Uppsala Astronomical Observatory. A.M.H.G. thanks the Mathematics and Statistics Department of the University of Newcastle-upon-Tyne and Instituto de Astrofísica de Andalucía. Finally, an anonymous referee is thanked for many valuable comments and suggestions which have improved the quality of this paper. This research made use of the NASA/IPAC Extragalactic Database (NED) which is operated by the Jet Propulsion Laboratory, California Institute of Technology, under contract with the National Aeronautics and Space Administration. 


\section{References}

Aller, L. H. 1984, Physics of the gaseous nebulae (Reidel Publishing Co., Dordrecht)

Baldwin, J. A., Phillips, M. M., \& Terlevich, R. 1981, PASP, 93,5

Belley, J., \& Roy, J.-R. 1992, ApJS, 78, 61

Bergvall 1999, private communication

Brocklehurst, M. 1971, MNRAS, 153, 471

Campbell, A., Terlevich, R., \& Melnick, J. 1986, MNRAS, 223, 811

Caplan, J., \& Deharveng, L. 1986, A\&A, 155, 297

Cerviño, M., \& Mas-Hesse, J. M., 1994, A\&A, 284, 749

Cohen, I. G., \& Blakeslee, J. P. 1998, AJ, 115, 2356

Copetti, M. V. F., Pastoriza, M. G., \& Dottori, H. A. 1986, A\&A, 156, 111

Costero, R., \& Peimbert, M. 1970, Bol. Obs. Ton. Tac., 5, 229

Dinerstein, H. L. 1990, The interstellar medium in galaxies, ed. Thronson, \& Shull, 257

Eskridge, P. B., \& White, R. E. III. 1997, AJ, 114, 988

Ferguson, A. M. N., Gallagher, J. S., \& Wyse, R. F. G. 1998, AJ, 116,673

Fillipenko, A. V. 1982, PASP, 94, 71

Gallagher III, J. S., Hunter, D. A., Gillett, F. C., \& Rice, W. 1991, ApJ, 371, 142

Gallart, C., Aparicio, A., Bertelli, G., \& Chiosi, C. 1996, AJ, 112,2596

Garnett, D. R. 1989, ApJ, 345, 282

Garnett, D. R. 1990, ApJ, 363, 142

González-Delgado, R. M., Perez, E., Tenorio-Tagle, G., et al. 1994, ApJ, 437, 239

Hidalgo-Gámez, A. M., \& Olofsson, K. 1998, A\&A, 334, 45

Hidalgo-Gámez, A. M., Olofsson, K., \& Masegosa, J. 2001, A\&A, submitted (Paper II)

Hodge, P., Lee., M. G., \& Kennicutt, R. C. Jr. 1989, PASP, 101, 32

Hodge, P., \& Miller, B. 1995, ApJ, 451, 176

Howarth, I. D., \& Lynas-Gray, A. E. 1989, MNRAS, 240, 513

Israel, F. P., Bontekoe, Tj. R., \& Kester, D. J. M. 1996, A\&A, 308, 723

Israel, F. P. 1997, A\&A, 317, 299

Izotov, Y. I., Thuan, T. X., \& Lipovetsky, V. A. 1997, ApJS, 108,1

Izotov, Y. I., \& Thuan, T. X. 1999, ApJ, 511, 639

Kinman, T. D., \& Davidson, K. 1981, ApJ, 243, 127

Kobulnicky, H. A., \& Skillman, E. D. 1996, ApJ, 471, 211

Kobulnicky, H. A., Skillman, E. D., Roy, J.-R., Walsh, J. R., \& Rosa, M. R. 1997, ApJ, 477, 679

Kurucz, R. L. 1992, The Stellar populations in Galaxies, ed. B. Barbuy, \& A. Renzini (Kluwer Academic Publishers), 225

Lequeux, J., Rayo, J. F., Serrano, A., Peimbert, M., \& Torres-Peimbert, S. 1979, A\&A, 80, 155
Liu, X.-W., \& Danziger, I. J. 1993, MNRAS, 263, 256

Liu, X.-W., Barlow, M. J., Danziger, I. J., \& Storey, P. J. 1995, ApJ, 450, L59

Longo, G., Ceriello, A., \& Capaccioli, M. 1991, A\&AS, 90, 375

Maeder, A. 1991, Publications de L'Observatoire de Geneve, No. 84

Marconi, G., Tosi, M., Greggio, L., \& Focardi, P. 1995, AJ, 109, 173

Masegosa, J. 1988, Ph.D. Thesis, Universidad de Granada

Masegosa, J., Moles, M., \& del Olmo, A. 1991, A\&A, 249, 505

Masegosa, J., Moles, M., \& Campos-Aguilar, A. 1994, ApJ, 420,576

McCall, M. L., Rybski, P. M., \& Shields, G. A. 1985, ApJS, 57,1

McGaugh, S. S. 1994, ApJ, 426, 135

Olofsson, K. 1995, A\&AS, 111, 570

Olofsson, K. 1997, A\&A, 321, 29

Osterbrock, D. E. 1989, Astrophysics of gaseous nebulae and active galactic nuclei (University Science Books, Mill Valley, CA)

Pagel, B. E. J., Edmunds, M. G., \& Smith, G. 1980, MNRAS, 193, 219

Pagel, B. E. J., \& Edmunds, M. G. 1981, ARA\&A, 19, 77

Pagel, B. E. J., Simonson, E. A., Terlevich, R. J., \& Edmunds, M. G. 1992, MNRAS, 233, 325

Peimbert, M. 1967, ApJ, 150, 825,

Peimbert, M., \& Spinrad, H. 1970, A\&A, 7, 311

Peimbert, M., \& Torres-Peimbert, S. 1977, ApJ, 168, 413

Petitpas, G. R., \& Wilson, C. D. 1998, ApJ, 496, 221

Rola, C., \& Pelat, D. 1994, A\&A, 287, 676

Roy, J.-R., \& Kunth, D. 1995, A\&A, 294, 432

Salpeter, E. E. 1955, ApJ, 121, 161

Saurer, W. 1998, MNRAS, 229, 51

Savage, B. D., \& Mathis, J. S. 1979, ARA\&A, 17, 73

Shield, G. A. 1990, ARA\&A, 28, 525

Schild, R. E. 1977, AJ, 82, 337

Skillman, E. D. 1984, Ph.D. Thesis, Washington University

Skillman, E. D., Terlevich, R., \& Melnick, J. 1989, MNRAS, 240,563

Skillman, E. D. 1989, ApJ, 347, 883

Stasińska, G. 1990, A\&AS, 83, 501

Talent, D. L. 1980, Ph.D. Thesis, Rice University

Tinsley, B. 1978, Fund. Cosm. Phys., 5, 287

Torres-Peimbert, S., \& Peimbert, M. 1977, Rev. Mex. Astron. Astrofis., 2, 181

de Vaucouleurs, G., de Vaucouleurs, A., Corwin, H. G., et al. 1991, Third Reference Catalogue of Bright Galaxies (Springer-Verlag)

Vila-Costas, M. B., \& Edmunds, M. G. 1993, MNRAS, 265, 199

Vílchez, J., \& Pagel, B. E. J. 1988, MNRAS, 231, 257

Whitford, A. E. 1958, AJ, 63, 201

Wilson, C. D. 1992, AJ, 104, 1374 